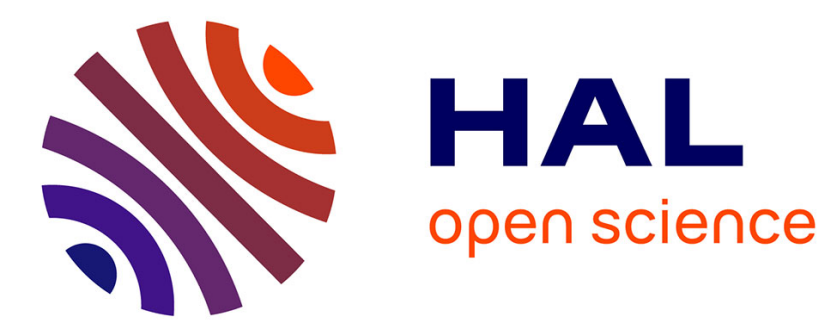

\title{
Scaling Analysis of the China France Oceanography Satellite Along-Track Wind and Wave Data
}

\author{
Yang Gao, François G Schmitt, Jianyu Hu, Yongxiang Huang
}

\section{To cite this version:}

Yang Gao, François G Schmitt, Jianyu Hu, Yongxiang Huang. Scaling Analysis of the China France Oceanography Satellite Along-Track Wind and Wave Data. Journal of Geophysical Research. Oceans, 2021, 126 (8), pp.e2020JC017119. 10.1029/2020JC017119 . hal-03319667

\section{HAL Id: hal-03319667 https://hal.science/hal-03319667}

Submitted on 12 Aug 2021

HAL is a multi-disciplinary open access archive for the deposit and dissemination of scientific research documents, whether they are published or not. The documents may come from teaching and research institutions in France or abroad, or from public or private research centers.
L'archive ouverte pluridisciplinaire HAL, est destinée au dépôt et à la diffusion de documents scientifiques de niveau recherche, publiés ou non, émanant des établissements d'enseignement et de recherche français ou étrangers, des laboratoires publics ou privés. 


\section{Scaling Analysis of the China France Oceanography SATellite Along-Track Wind and Wave Data}

Yang Gao ${ }^{1,2}$, Francois G Schmitt $^{2}$, Jianyu Hu ${ }^{1,3}$, Yongxiang Huang ${ }^{1,3,4,5}$

${ }^{1}$ State Key Laboratory of Marine Environmental Science, College of Ocean and Earth Sciences, Xiamen University, Xiamen 361102, China

${ }^{2}$ CNRS, Univ. Lille, Univ. Littoral Cote d'Opale, UMR 8187, LOG, Laboratoire d'Océanologie et de Géosciences, F 62930 Wimereux, France

${ }^{3}$ Southern Marine Science and Engineering Guangdong Laboratory (Zhuhai), Zhuhai 519000, China

${ }^{4}$ Fujian Engineering Research Center for Ocean Remote Sensing Big Data, Xiamen 361102, China ${ }^{5}$ SJTU SMSE-Mingguang Joint Research Center for Advanced Palygoskite Materials, Mingguang 239400,

China

\section{Key Points:}

- Fourier power spectra are retrieved for both wind and wave fields simultaneously measured by the China France Oceanography SATellite.

- Due to a large scale separation, the multiscale scaling features for both wind and wave fields are observed from 100 to $3000 \mathrm{~km}$.

- Meridional and seasonal variations of the scaling features are evident.

Corresponding author: Y.H., yongxianghuang@xmu.edu.cn 


\begin{abstract}
Turbulence or turbulence-like phenomena are ubiquitous in nature, often showing a powerlaw behavior of the fluctuations in either spatial or temporal domains. This power-law behavior is due to interactions among different scales of motion, and to the absence of characteristic scale. In this work, we consider the multiscale dynamics of China France Oceanography SATellite (CFOSAT) data as atmospheric and oceanic quantities influenced by turbulence. Fourier power spectra were estimated for the data provided by the CFOSAT via the Wiener-Khinchine theorem to extract multiscale information for both wind speed (WS) and significant wave-height (Hs). The WS data were collected from December 18, 2018 to August 31, 2020, and the Hs data from July 29, 2019 to August 31, 2020. Fourier power spectra for both WS and Hs exhibit power-law features in the ranges of $100 \mathrm{~km}$ to $3000 \mathrm{~km}$ with a scaling exponent $\beta$ varying from $5 / 3$ to 3 . The global distributions and seasonal variations of $\beta$ for both WS and Hs have also been considered. The results show that due to the energetic convective activities in the low-latitude zones, the scaling exponents $\beta$ in these regions are closer to the value of $5 / 3$. Concerning the seasonal variations, for most regions, the scaling exponents in winter are larger than those in summer for WS. The seasonal variations of $\beta$ in low-latitudes are stronger than those in the mid-latitudes. Our preliminary results enrich the fundamental knowledge of ocean surface processes and also provide a benchmark for either oceanic or atmospheric models.
\end{abstract}

\title{
Plain Language Summary
}

The China France Oceanography SATellite (CFOSAT) can measure simultaneously wind and wave, providing a unique data set to assess the coupling of the wind and wave during the air-sea interactions. Here we show that the multiscale spatial statistics is relevant for both wind and wave fields. More precisely, spatial scaling behavior is found on the spatial scale range of $100 \mathrm{~km}$ to $3000 \mathrm{~km}$, with a scaling exponent varying on different oceans and seasons. Furthermore, the dynamical coupling between the wind and wave is characterized by the Fourier-based co-spectrum and coherence function analysis. The results obtained in our study not only enrich our fundamental knowledge of ocean surface processes, but also provide a benchmark for either oceanic or atmospheric models.

\section{Introduction}

Ocean waves and wind play critical roles at various scales as they regulate the functioning of Earth's climate and weather system (Bigg et al., 2003). The momentum and heat exchanges between the ocean and the atmosphere are affected by waves and wind. For example, some aerosols are generated by wave breaking and transported by wind (Kunz et al., 2002); the growth and decay of sea ice are influenced by waves and wind (Schlosser et al., 2018); waves and wind also show critical interactions with surface currents and upper ocean turbulence (Babanin, 2006), to list a few. Furthermore, waves and wind are major information for the safety of offshore operations and the forecast of marine severe weather events. Therefore, waves and wind, especially significant wave height $(\mathrm{Hs})$ and wind speed (WS) are of interest for many fields from scientific researches to engineering applications.

The aims of studying the waves and wind are also to improve the ocean dynamic modeling and prediction, marine meteorology forecast, climate variability knowledge, and fundamental knowledge of surface processes (Hauser et al., 2016). Due to extremely high Reynolds numbers, the non-linearities and multiple spatial-temporal scales characterizing the ocean and atmosphere, it is hard to describe, simulate or predict their complicated motions precisely (Vallis, 2017). Most oceanic, atmospheric or global models are operated by averaging Navier-Stokes equations at large scales. Turbulent closures are needed to express the effects of small-scale fluxes to large scale processes, but exact expressions are still lacking (Pope, 2001; Schmitt, 2007; Egolf \& Hutter, 2020), indicating that oceanic and atmospheric models have still fundamental weaknesses. 
The foundation of studying the wind and waves comes from field observations. Relatively accurate measurements of wind speed were possible after the invention of the anemometer by Thomas Romney in 1846. Then, more precise wind speed observations have been achieved by the development of hot wire anemometers, while the wind could only be measured in a fixed point, until the occurrence of Doppler radars. For estimating the wind at a global scale, a satellite onboard wind scatterometer was developed during the Seasat mission in the 1970s. After that, a series of satellites which can observe the wind field have been launched, such as Nimbus-7 (1979), Advanced Earth Observing Satellite (ADEOS, 1996), Quick Scatterometer (QuikSCAT, 1999), Meteorological Operational Satellites (METOP-A, METOP-B, and METOP-C, mission since 2006), Cyclone Global Navigation Satellite System (CYGNSS, 2017), HaiYang-2B (HY-2B, 2018), to name a few. Before 1975, wave height data have been mainly collected by buoys or coastal marine observation stations. Since that year, global ocean wave heights could be retrieved by satellites equipped with radar altimeters, such as for instance, Geodetic Earth Orbiting Satellite-3 (GEOS-3, 1975), European Remote-Sensing Satellite (ERS-1, 1991), Topography Experiment/Poseidon (TOPEX, 1996), JASON-1 (2001), and followed by JASON-2 (2008), and JASON-3 (2016), HaiYang2A (HY-2A, 2011), to list a few.

Previously, due to the limitation in the available data, the dynamic analysis of wind and waves have mostly been done separately. Since the ocean surface waves are most commonly driven by wind through frictions between wind and surface waters, the energy and momentum of waves are injected by the wind blowing over the sea surface. In some light WS regions, such as in the tropics, the energy and momentum can also be transferred back from waves to wind (Hanley et al., 2010). To determine the dynamical coupling between wind and waves, a detailed study of the multiscale properties of wind and waves is required. Thanks to the China France Oceanography SATellite (CFOSAT), the WS and Hs data can be observed simultaneously from space for the first time. The CFOSAT project provides a unique chance to analyze the wind and waves together at a global scale.

In this study, on the basis of the WS and Hs data observed by CFOSAT, Fourier power spectra are estimated via the Wiener-Khinchine theorem to study scaling properties and evaluate scaling ranges and slopes. In addition, the spatial and temporal variations of the scaling exponents are examined. In the following, firstly some theories for atmospheric wind scaling regimes are recalled, as well as for the oceanic waves. Then the data and methods are presented, followed by a section presenting the results, and two last sections are devoted to discussions and conclusions.

\section{Theories}

\subsection{Theories for the Atmospheric Wind}

Assuming that there are no characteristic length scales, over given scale ranges, several scaling laws have been proposed for the wind field in the atmosphere. Here these theoretical proposals are recalled briefly. They will be used as comparisons and potential explanations when considering the CFOSAT data analysis in the following sections.

The most classical scaling is Kolmogorov's 1941 assumption of universal fluctuations of the turbulent wind velocity in the inertial range, using locally isotropic and homogeneous hypotheses. For very large Reynolds numbers, as is the case in the atmosphere, one obtains (Kolmogorov, 1941; Obukhov, 1941):

$$
E_{u}(k)=C_{0} \epsilon^{-2 / 3} k^{-5 / 3},
$$

where $E_{u}(k)$ is the Fourier power spectrum of the wind velocity, $\epsilon$ is the mean energy dissipation rate per unit mass, $k$ is one component of the wavenumber vector $\mathbf{k}=\left(k_{x}, k_{y}, k_{z}\right)$, and $C_{0}$ is a constant. In case of intermittency there may be some small corrections to the value of the spectral slope $-5 / 3$, with slopes that may be slightly steeper, e.g., an 
experimental value of -1.70 has been reported (Monin \& Yaglom, 1971; Frisch, 1995). This framework corresponds to a cascade of energy as proposed by Richardson in the 1920s (Richardson, 1922): the energy is injected at large scales, is cascading in the inertial range and is dissipated at small scales, smaller than the Kolmogorov scale, which is of the order of millimeters. The Reynolds number in the atmosphere is in the order of $10^{7}$ to $10^{9}$, and the $-5 / 3$ slope has been found from atmospheric observations by many authors (Sreenivasan \& Antonia, 1997; Calif \& Schmitt, 2012; Schmitt \& Huang, 2016).

The atmosphere is stratified and at very large scales the atmospheric motions seem to be quasi-two dimensional (Danilov \& Gurarie, 2000). This motivated the application of 2D turbulence phenomenology, as developed by Kraichnan (1967). The phenomenology of 2D turbulence is very different from $3 \mathrm{D}$ turbulence. There is no vortex-stretching term, and the enstrophy (square of vorticity) can be introduced to propose an enstrophy cascade picture (Wyngaard, 2010): enstrophy is produced at large scales through mean vorticity gradient, and is destructed at small scales by viscosity. The enstrophy spectrum has dimensionally a -1 slope, and since this is $k^{2}$ times the energy spectrum, in this range of scales the energy spectrum follows (Kraichnan, 1967):

$$
E_{u}(k)=C_{1} \zeta^{2 / 3} k^{-3}, k>k_{f}
$$

where $C_{1}$ is a constant, $\zeta$ represents the dissipation rate of enstrophy, and $k_{f}$ is the wavenumber corresponding to the forcing. At this forcing scale, the enstrophy goes down to small scales, and the energy has an inverse cascade, going to large scales, with a scaling range as (Kraichnan, 1967; Vallis, 2017):

$$
E_{u}(k)=C_{2} \epsilon^{2 / 3} k^{-5 / 3}, k<k_{f}
$$

At very large scales, the energy is assumed to be dissipated by friction through Ekman layers. For the application of this approach to the atmosphere, a quasigeostrophic theory has been proposed (Charney, 1971), where the enstrophy is replaced by a pseudo potential enstrophy. In such framework, contrary to Kraichnan's 1967 model, there is a -3 slope at large scales and a $-5 / 3$ slope at small scales, for the power spectrum of the velocity field.

Finally, let us also mention the 23/9D model proposed by Schertzer and Lovejoy since the 1980s (Schertzer \& Lovejoy, 1985, 1987; Lovejoy et al., 2009). Anisotropic feature are suggested at all scales with different scaling exponents in the horizontal and vertical directions, where the former is dominated by the energy flux with a slope of $-5 / 3$ and the latter by the buoyancy variance flux with a slope $-11 / 5$ (Bolgiano, 1959; Obukhov, 1959). Later, the horizontal anisotropy has been taken into account in the 23/9D model and verified by using the European Centre for Medium-Range Weather Forecasts (ECMWF) reanalyses data (Lovejoy \& Schertzer, 2011). This model is also discussed and checked in more recent works (Lovejoy \& Schertzer, 2013; Pinel \& Lovejoy, 2014). The results show that this model is capable to explain numerous claims of transition phenomena, for instance the spurious -2.4 slope for aircraft collected data can be interpreted by the anisotropic 23/9D turbulence model, rather than the isotropic $3 \mathrm{D}$ or $2 \mathrm{D}$ turbulence models.

\subsection{Theories for the Oceanic Waves}

Under a turbulent wind forcing over the sea, a part of the wind energy is transferred to the water masses through the surface wind shear. Since the wind forcing, in the inertial range, has no characteristic scale, and since the related processes involved no obvious characteristic scales, scaling laws for the sea surface height (SSH) have been proposed since the 1950s. They write as:

$$
E_{\Psi}(k) \sim k^{-B}
$$

where $E_{\Psi}(k)$ is the Fourier power spectrum of the spatial 2D SSH field, and $B$ is the scaling exponent. This is valid in a range found over high wavenumbers corresponding to a socalled equilibrium range involving gravity waves. Such range describes breaking waves have 
large curvatures, and even nearly discontinuous slopes, with sharp and random crests. By considering dimensional analysis, one early proposal was Phillips (1985a), who proposed $B=4$. This value was also advanced later by Belcher and Vassilicos (1997) using different dynamical and geometrical arguments: $B=5-D$ was proposed. With considering the balance arguments, a value of the dimension $D=1$ was found. On the other hand Phillips (1985b) revoked the $B=4$ result and proposed $B=7 / 2$, for taking into account wave breaking and wave-wave interactions, and assuming that the nonlinear energy flux, wind forcing, and dissipation are in balance, proportional, and of comparable magnitude.

These results concern about the SSH variable, at small scales, in part smaller than the scale range spanned by satellites. What is recorded by CFOSAT satellite is $\mathrm{Hs}$, a local indicator, at the pixel size, of the intensity of the waves. The Hs has been originally defined as the average of the highest one-third of waves that occur during a given period. Nowadays it is usually defined as four times the standard deviation of the ocean surface elevation. As the value of Hs is estimated locally, it may have also spatial variations. However, its relation with SSH is not direct and there is no theoretical predictions in the literature concerning a scaling behavior of Hs, either directly or in relation with theoretical proposals for SSH.

\section{Data and Methods}

\subsection{Data}

The simultaneously observed WS and Hs data in this study are provided respectively by a rotating fan beam scatterometer (SCAT) and surface waves investigation and monitoring radar (SWIM), both boarded on CFOSAT. Both instruments use $K u$ band microwave frequencies, and SCAT uses medium incidence angles (from $26^{\circ}$ to $46^{\circ}$ ) to retrieve the wind vectors at $10 \mathrm{~m}$ height, whereas SWIM operates at near-nadir incidence angles (from $0^{\circ}$ to $10^{\circ}$ ) to retrieve sea-surface waves (Liu et al., 2020). With an orbital repetition cycle of 13 days and accounting for the instrument geometry, which can provide a $1000 \mathrm{~km}$ width of swath for the wind field as illustrated in Figure 1a, the system provides a global coverage within 3 days for wind fields and nearly global for waves (Hauser et al., 2016).

The wind field is not directly measured; it is estimated using a geophysical model function, which gives the relation between the microwave scattered field, incidence angle, wind direction and speed close to the sea. Using this approach, an algorithm is designed to retrieve the $10 \mathrm{~m}$ wind intensity and direction, from backscattered data from the SCAT instrument. When the scatterometer has an inclined beam, both wind speed and direction can be retrieved. This is why rotating beam scatterometers are used since SeaWinds onboard the satellite QuikSCAT (Spencer et al., 1997). A similar design is used in CFOSAT, using two fan beams, one vertically polarized, and the other horizontally polarized (Lin \& Dong, 2011; Lin et al., 2018). The processing done involves averaging several backscatter values having similar incidence and azimuth angles: the larger the number of views, the more precise results can be obtained. As compared to fixed fan beams or pencil beams scatterometers, the rotating fan beam system used by SCAT can obtain more observations of the azimuth angles within a single swath: normally 4-16, more than ten in most areas for SCAT, while for fixed fan beams or pencil beams, the maximum number is equal to 4 (Zhang et al., 2021). Thus, the wind information retrieval accuracy is greatly increased in the CFOSAT mission (Liu et al., 2020).

For obtaining Hs, an "adaptive retracking" algorithm is performed (Tourain et al., 2021). The main idea of this algorithm is the following. Different pulses are received by the satellite sensor, coming from reflections at the ocean's surface. These different backscattered echos are classically treated using a model of the ocean's rough surface (Brown, 1977), seen as the convolution of a point source, a flat sea surface and an assumed probability density function of sea elevation. Different improvements of this algorithm have been proposed since, and a modified version, used for CFOSAT, is described in details in Tourain et al. (2021). 
This algorithm is still based on the fit of the Brown model echo (Brown, 1977) to the recorded waveforms and the use of a maximum likelihood estimator. One of the improvements as compared to the conventionally used algorithms for other altimeter missions is the use of the real point target response from the SWIM sensor, instead of a theoretical one. Other improvements are linked with inversion methods using new analytical models.

With all of these improvements, the performance of the CFOSAT mission was found to be remarkable: the accuracy of the Hs is $25-30 \mathrm{~cm}$ or $5 \%$ of the mean value; the accuracy of the wind filed is $2 \mathrm{~m} / \mathrm{s}$ in magnitude and $20^{\circ}$ in direction (Suquet et al., 2019; Liu et al., 2020; Hauser et al., 2020; Li et al., 2021).

The WS data used in this study are in a $12.5 \mathrm{~km}$ resolution covering the period from December 18, 2018 to August 31, 2020, which is nearly 9200 orbits. For each orbit, the satellite can generate a wind field dataset with a size of $84 \times 3440$ pixels. Conventionally, the measured WS is treated as 10 meter wind above the sea surface. In other words, it is still in the marine-atmospheric boundary layer. The Hs data analyzed here were collected from July 29, 2019 to August 31, 2020, which is 7200 orbits with a nominal spatial resolution of 1.5 $\mathrm{km}$. Figure 1a shows an example of the along-track wind field and Hs data collected in the western Pacific Ocean on September 4, 2019 during the Typhoon Lingling (Xu et al., 2019). The corresponding along-track WS and $\mathrm{Hs}$ are shown as black and red dots respectively in Figure 1b. The signal of Typhoon Lingling (2019) can be distinguished by the CFOSAT observation, and the typhoon-induced enhancements of WS and Hs are clearly visible. Note that the WS and Hs data used in this paper are Level-2 products, which means the data are processed without any further interpolations. Thus, the original dynamical features of atmosphere and ocean surface can be well preserved in these data.

Before the processing of the data, quality control (QC) was performed. For WS, only the data collected by more than two beams are considered. As for Hs, a Hampel identifier (Davies \& Gather, 1993) is chosen to detect the outliers. Hampel identifier uses the median and median absolute deviation as a robust estimate of the location and spread of the outliers. For each data series, firstly a value of $W$ points of the window half-width is given. Then the identifier computes the median of a window composed of the sample and its $2 W$ surrounding samples, $W$ data points per side. Besides, the median absolute deviation is also estimated. If a sample differs from the median by more than three times the standard deviations, it is treated as an outlier. This identifier has been proven extremely effective in practice for various fields (Pearson, 2002; Pearson et al., 2015, 2016). In this study, the window halfwidth is set as 150 data points and the abnormal data are set as Not-a-Number (NaN). As a result, there are roughly $15 \%$ of the WS and $2 \%$ of the Hs data discarded.

Figure 2 shows an example of the WS and Hs data before and after QC. The black dots represent the raw data provided by the CFOSAT, and the red dots are the data after QC. To minimize the estimation bias for the calculated Fourier power spectrum, a small portion of normal data which successive to the abnormal ones are also excluded in the $\mathrm{QC}$ procedure. Figure 3 shows the probability density functions (pdf) of WS and Hs before and after QC, computed over all the data: the pdf tails are cut off after QC. The pdf of WS shows an exponential tail for values larger than $16 \mathrm{~m} / \mathrm{s}$. The tail is in the form of $\exp \left(-x / V_{0}\right)$ where the characteristic scale is $V_{0}=1 / 0.31=3.22 \mathrm{~m} / \mathrm{s}$. For Hs data, there is also an exponential form in the ranges of 5 to $15 \mathrm{~m}$ : here the tail is in the form of $\exp \left(-x / L_{0}\right)$ where the characteristic scale is $L_{0}=1 / 0.39=2.56 \mathrm{~m}$.

\subsection{Spectra and Co-spectra Estimated via the Wiener-Khinchine Theorem}

In this work, the Fourier power spectra $E(\mathbf{k})$ are estimated via the Wiener-Khinchine theorem. More precisely, the Fourier transform of the autocorrelation functions $\rho(\mathbf{r})$ as follows:

$$
E_{\phi}(\mathbf{k})=\mathcal{R} \int_{-\infty}^{+\infty} \widetilde{\rho}(\mathbf{r}) \exp (-j 2 \pi \mathbf{k r}) d \mathbf{r}
$$



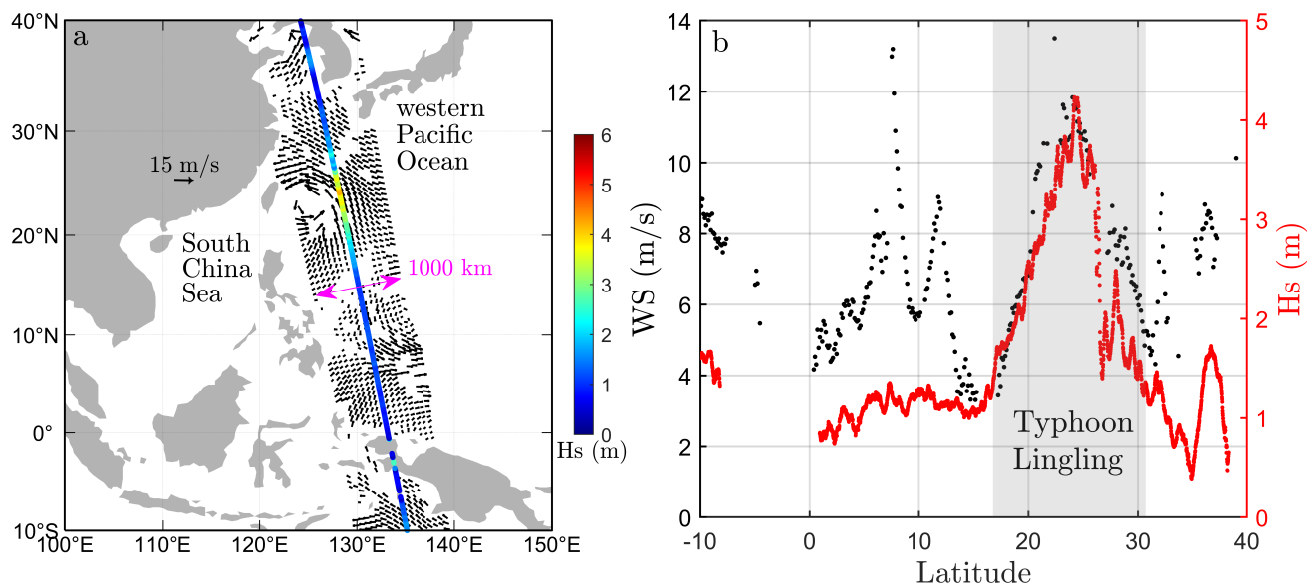

Figure 1. (a) Simultaneously observation of wind vectors (black arrows) and Hs (color dots) by CFOSAT in the western Pacific Ocean on September 4, 2019 during the Typhoon Lingling. (b) The corresponding along-track WS observed by SCAT (black dots) and Hs observed by SWIM (red dots).
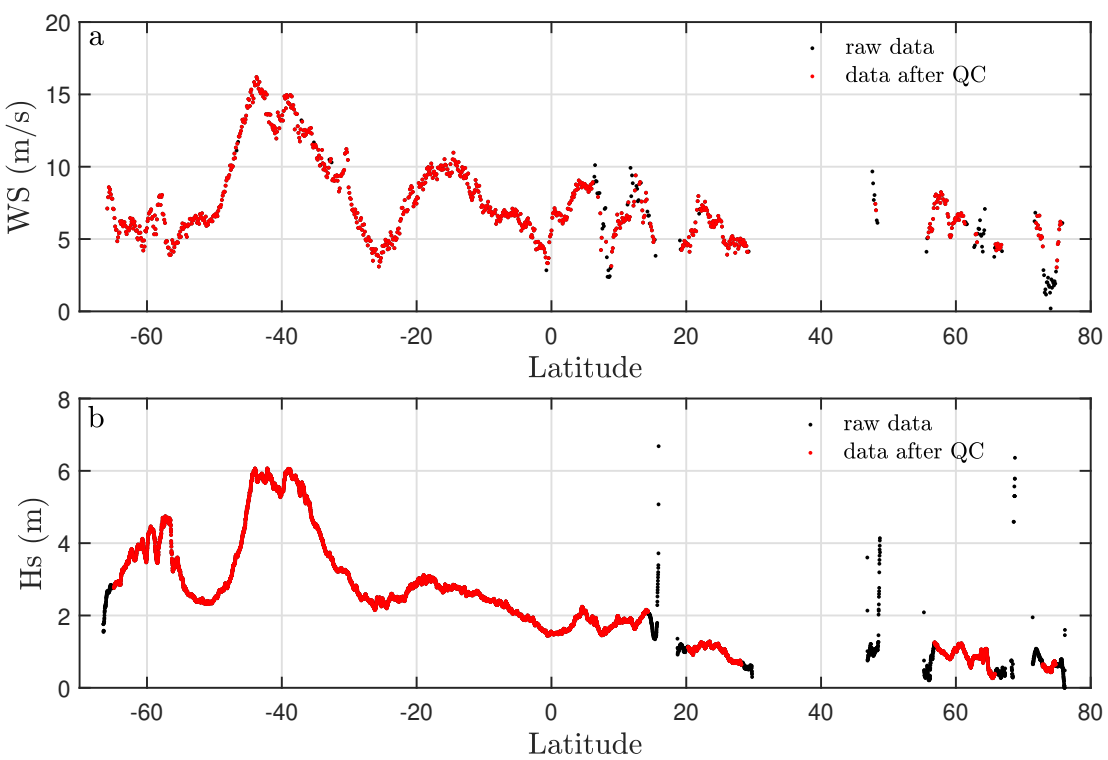

Figure 2. Comparisons of raw data (black dots) and the data after quality control (red dots). (a) and (b) indicate WS and Hs data, respectively. 

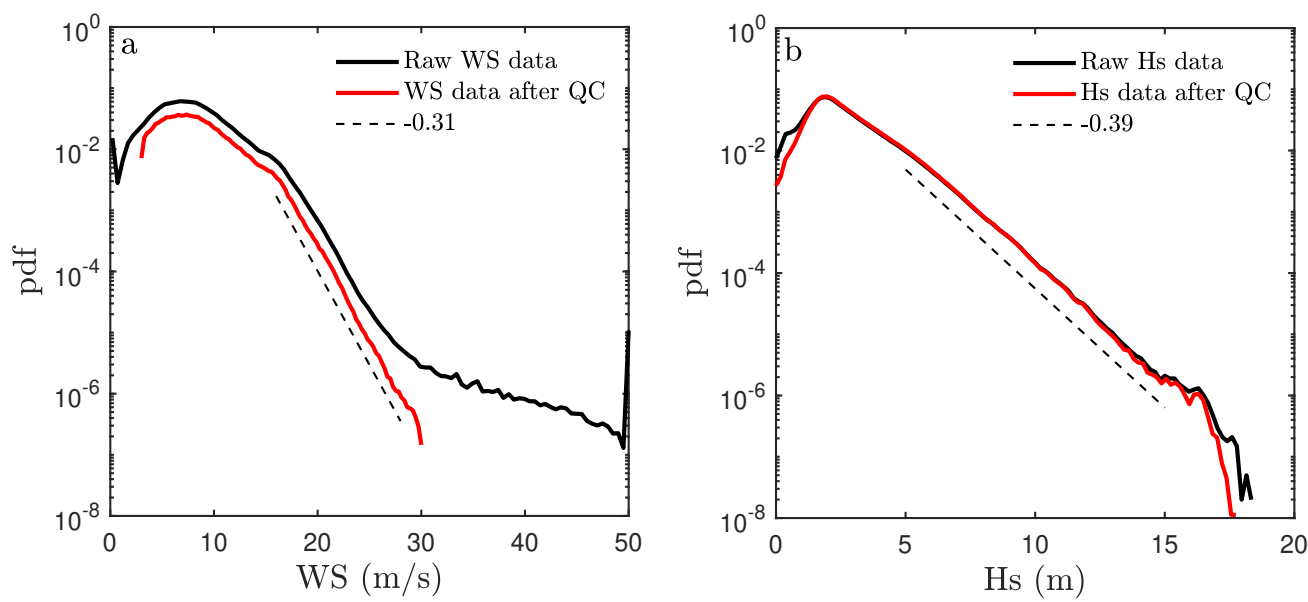

Figure 3. The comparisons of the pdfs of WS and Hs data before (black curves) and after (red curves) QC. Dashed lines emphasize the exponential tails which are found, and the fitted slopes are indicated.

in which $\mathcal{R}$ means real part, $j=\sqrt{-1}$ is the complex unit, $\mathbf{k}$ is the wavenumber vector, $\mathbf{r}$ is the distance vector, and,

$$
\widetilde{\rho}(\mathbf{r})=\left\langle\rho_{m}(\mathbf{r})\right\rangle_{m}, \quad \rho_{m}(\mathbf{r})=\frac{1}{M_{m}(\mathbf{r})} \sum_{i=1}^{M_{m}(\mathbf{r})} \widetilde{\phi}_{m}\left(\mathbf{x}_{i}+\mathbf{r}\right) \widetilde{\phi}_{m}\left(\mathbf{x}_{i}\right)
$$

where $\widetilde{\phi}_{m}\left(\mathbf{x}_{i}\right)=\phi_{m}\left(\mathbf{x}_{i}\right)-\left\langle\phi_{m}\left(\mathbf{x}_{i}\right)\right\rangle$ is the centered $\phi_{m}\left(\mathbf{x}_{i}\right), \phi_{m}$ is the value of either WS or Hs of the $m$ th orbit, \langle\rangle$_{m}$ means spatial average over the $m$ th orbit, and $M_{m}(\mathbf{r})$ is the sample size at the separation scale $r$ for the $m$ th orbit. Due to several reasons, such as the presence of sea ice, lands, bad measurements, the collected WS and Hs data often contain gaps. The use of the autocorrelation here prevents problems coming from small gaps. To mitigate the impacts of missing data to the spectral analysis, orbits with the ratio of more than $50 \%$ of the valid data are then accepted. It is found empirically that the averaged autocorrelation function $\widetilde{\rho}(\mathbf{r})$ and the corresponding power spectra $E_{\phi}(\mathbf{k})$ are not affected by these missing data. In this work, the radially averaged Fourier power spectrum of the WS data is also estimated: in such case, the autocorrelation function of the $2 \mathrm{D}$ wind field is estimated; then the $2 \mathrm{D}$ power spectrum $E_{u}(\mathbf{k})$ is calculated via the Wiener-Khinchine theorem. Assuming a horizontal statistical isotropy, the radially averaged Fourier power spectrum $E_{u}(\hat{k})$ is estimated by an angle integration of the $2 \mathrm{D}$ spectrum. For a convenient comparison with the 1D spectrum, the final radially averaged spectrum is multiplied by the wavenumber modulus $\hat{k}=|\mathbf{k}|$.

In case of scaling and isotropy, a power-law behavior of the Fourier spectrum is expected:

$$
E_{\phi}(k) \propto k^{-\beta},
$$

where $k$ is one component of the wavenumber vector $\mathbf{k}$, and $\beta$ is the so-called scaling exponent, which represents the absolute value of the slope of the best-fit straight line between $\log \left(E_{\phi}(k)\right)$ and $\log (k)$ on a certain range of $k$ values corresponding to the scaling range. Let us note that the Hs data are scalar, whereas WS data are $2 \mathrm{D}$ vectors. In the remaining of the manuscript, the WS analysis is done on the velocity amplitude, except when zonal and meridional components of WS are compared: in the latter case each vectorial component is analyzed separately for comparison.

For estimating the co-spectrum for WS and Hs, we perform a similar procedure. The mean cross-correlation function $\widetilde{\rho}_{W S, H s}(\mathbf{r})$ is estimated. The co-spectrum is then derived 
from Fourier transform via the Wiener-Khinchine theorem:

$$
E_{W S, H s}(\mathbf{k})=\mathcal{R} \int_{-\infty}^{+\infty} \widetilde{\rho}_{W S, H s}(\mathbf{r}) \exp (-2 j \pi \mathbf{k r}) d \mathbf{r} .
$$

\subsection{Coherency Spectrum}

To identity the relation between WS and Hs, the co-spectrum is normalized with the 1D spectra for WS and Hs to get the coherency spectrum,

$$
H_{W S-H s}(\mathbf{k})=\frac{\left|E_{W S, H s}(\mathbf{k})\right|^{2}}{E_{W S}(\mathbf{k}) E_{H s}(\mathbf{k})}
$$

where $0 \leq H_{W S-H s}(\mathbf{k}) \leq 1$. When the value of coherence function value is equal to 1 , there is a perfect linear relationship between the two signals. When the value is 0 , then there is no relationship between the two signals (Formenti, 1999).

\subsection{De-aliasing}

Another problem often occurring in the Fourier power spectrum analysis is due to aliasing: when the sampling frequency rate is less than twice the highest frequency of the physical process, namely, the data are undersampled. For undersampled datasets, measurements of their power spectra will often be distorted by aliasing, not only near the Nyquist frequency, but also below it (Kirchner, 2005). To correct the distortions introduced by spectral aliasing, and recover the spectrum, Kirchner (2005) proposed a filtering method which does not require some a priori knowledge of the true spectrum. Here note $E_{X}(f)$ is the true spectrum and $E_{Y}(f)$ is the measured one which includes some degree of aliasing. The formula to derive $E_{X}(f)$ from $E_{Y}(f)$ is as follows,

$$
E_{X}(f)=\frac{E_{X_{\text {model }}}(f)}{E_{Y_{\text {model }}}(f)} E_{Y}(f),
$$

in which $E_{X_{\text {model }}}(f)$ and $E_{Y_{\text {model }}}(f)$ are model functions defined as

$$
E_{X_{\text {model }}}(f)=\frac{E_{0} f^{-\alpha}}{1+\left(f / f_{c}\right)^{2}} \approx\left\{\begin{array}{l}
E_{0} f^{-\alpha}, f \ll f_{c} \\
E_{0} f_{c}^{2} f^{-(\alpha+2)}, f \gg f_{c},
\end{array}\right.
$$

and

$$
E_{Y_{\text {model }}}(f)=E_{X_{\text {model }}}(f)+\sum_{n=1}^{\infty} E_{X_{\text {model }}}\left(n f_{s}-f\right)+E_{X_{\text {model }}}\left(n f_{s}+f\right) .
$$

Here $E_{0}$ is an arbitrary constant that sets the scale of the spectral power, $f_{s}$ is the sampling frequency and $f_{c}$ indicates the corner frequency, which must typically be specified a priori. As long as $f_{c}$ is substantially above $f_{s}$, the exact value of $f_{c}$ will have little effect on the aliasfiltered spectrum. The parameter $\alpha$ is auto determined by a nonlinear fitting procedure, which finds the value of $\alpha$ for which $E_{Y_{\text {model }}}(f)$ matches the measured spectrum $E_{Y}(f)$ as closely as possible. In this way, the effects of aliasing to the spectral analysis can be suppressed.

\section{Results}

\subsection{Scaling Features at the Global Scale}

The global averaged spectra of WS (blue curves) and Hs (red curves) are shown in Figure 4. The dotted and solid curves indicate the raw and alias-filtered spectra, respectively. The straight lines are given as references with different slopes. In the high wavenumber ranges, due to the existence of spectral aliasing, the raw spectra exhibit upward trend, mainly visible for WS. After applying the alias-filtered processes, the tails are downward 


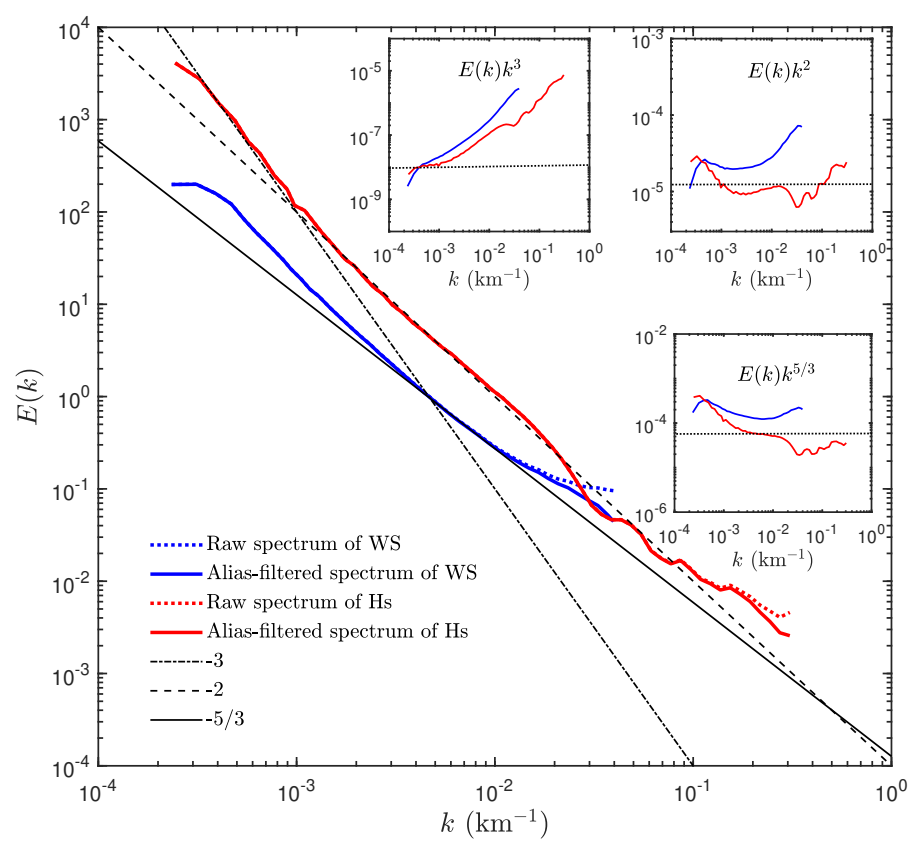

Figure 4. The global averaged Fourier power spectra of WS (blue curves) and Hs (red curves), the dotted and solid line styles mean the raw spectra and the spectra after alias-filtered. The black dash dotted, dashed, and thin lines are given as references with the slopes of $-3,-2$, and $-5 / 3$. The insets show the corresponding compensated spectra.

recovered. In the wavenumber ranging from $1 \times 10^{-3}$ to $1 \times 10^{-2} \mathrm{~km}^{-1}$, corresponding to the spatial scales $(r)$ from 100 to $1000 \mathrm{~km}$, the scaling exponent $\beta$ for $\mathrm{Hs}$ is close to 2, as shown in Figure 4. For wavenumbers smaller than $1 \times 10^{-3} \mathrm{~km}^{-1}(r \geq 1000 \mathrm{~km}), \beta$ for Hs approaches 3. As for the wavenumbers higher than $1 \times 10^{-2} \mathrm{~km}^{-1}(r \leq 100 \mathrm{~km})$, the spectrum of Hs illustrates a conspicuous decreasing trend in the spatial scales from 30 to 100 $\mathrm{km}$, then small fluctuations occur along the spectrum. The spectrum of WS also exhibits power-law features in the range from 100 to $3000 \mathrm{~km}$, and the values of $\beta$ are between $5 / 3$ and 3 with small variations. A finer inspection shows that in the range from $100 \mathrm{~km}$ to 250 $\mathrm{km}$, the slopes of WS spectra are close to $-5 / 3$. These multiscale features for WS and $\mathrm{Hs}$ are also emphasized in the compensated spectra as insets.

The averaged spectra of zonal and meridional components of WS are also calculated to tentatively test the horizontal directional isotropy of the wind field. The comparison of these two spectra is illustrated in Figure 5a. The blue and red curves represent the zonal and meridional component along track spectra, respectively. These two spectra show similar scaling features, with the zonal component spectrum slightly larger than the meridional one, which has been previously reported by Freilich and Chelton (1986). In order to better compare these two spectra, we introduce their ratio as:

$$
I\left(k_{y}\right)=\frac{E_{v}\left(k_{y}\right)}{E_{u}\left(k_{y}\right)},
$$

where $v$ and $k_{y}$ are the velocity and the wavenumber components along track. This ratio is displayed in Figure $5 \mathrm{~b}$. The inset in log-log plot shows a horizontal line, confirming that zonal and meridional spectra have the same scaling exponent, from 100 to $3000 \mathrm{~km}$. In turbulence such ratio is called the isotropy ratio and for $5 / 3$ spectra, in case of isotropy, the value $I\left(k_{x}\right)=3 / 4$ is derived, using symmetry arguments, from the continuity equation (Kolmogorov, 1941; Monin \& Yaglom, 1971). 

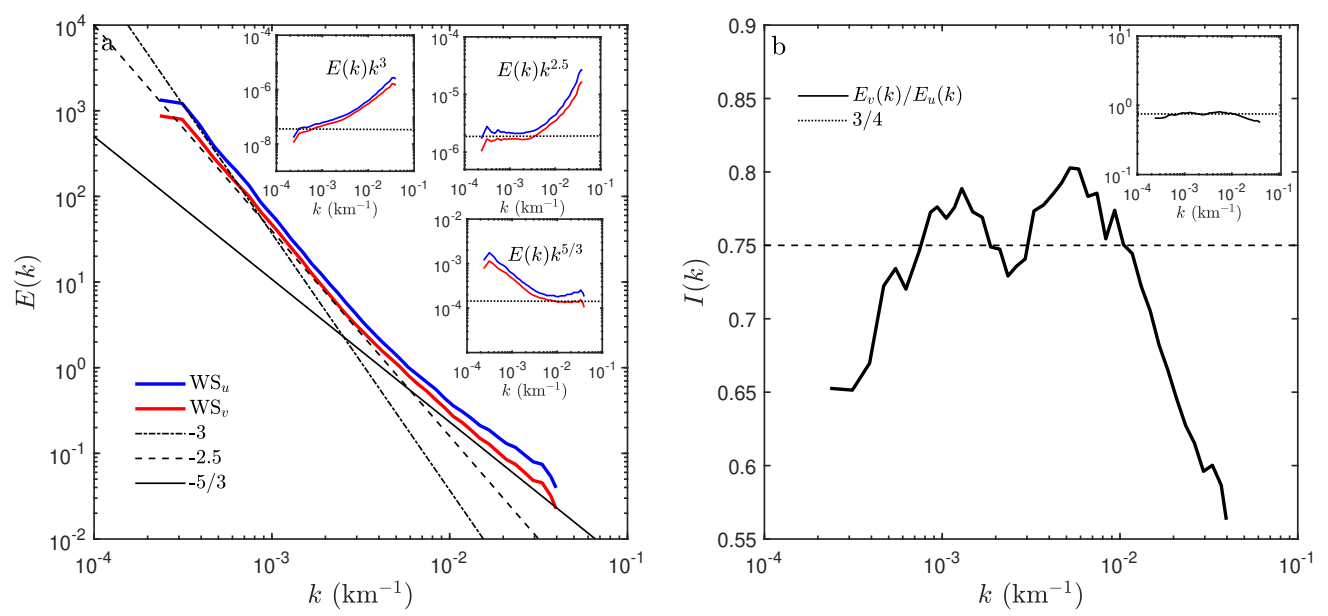

Figure 5. (a) The global averaged Fourier power spectra of zonal (blue curve) and meridional (red curve) components of WS; the black lines are references. The insets show the corresponding compensated spectra. (b) The corresponding isotropy ratio function; the dashed black line indicates the theoretical value 3/4 expected in case of isotropy (Kolmogorov, 1941); the inset is in log-log plot.

To consider the dynamical links between WS and Hs, the co-spectrum $E_{c o}(k)=$ $E_{W S, H s}(k)$ for WS and Hs is estimated and illustrated in Figure 6 a. Power-law features can be found from the corresponding spatial ranges from 30-250 km and 250-2000 km with the scaling exponents equal to 1.8 and 2.4, respectively. The compensated spectra as inset confirm the scaling features. The coherence function $H_{W S-H s}(k)$ between WS and Hs is also estimated and shown in Figure 6b, where a log-log plot is shown as inset. The values of coherence function lie between 0.04 and 0.13 in the whole wavenumber domain, with a peak value occurring in the separation scale around $1200 \mathrm{~km}$.

\subsection{Analyses in Basin Scales}

In order to perform more analyses at smaller portions of the globe, the world ocean is separated into five regions: the Indian Ocean, the North Atlantic Ocean, the South Atlantic Ocean, the North Pacific Ocean, and the South Pacific Ocean. This provides scaling characteristics of WS and Hs spectra in different oceanic areas. The measured ensemble averaged Fourier power spectra are shown in Figure 7a (WS) and Figure 7b (Hs). The spectra measured from different ocean basins have all the same shape and share the same scaling properties.

The spectra of WS display a very clear scaling with exponents close to 2 , for scales from 25 to $2500 \mathrm{~km}$, for all basins. Concerning $\mathrm{Hs}$, the scaling exponents $\beta$ are close to 3 , and 2 for the ranges from 500 to $3000 \mathrm{~km}$ and 50 to $500 \mathrm{~km}$, respectively. The compensated spectra are also given in Figure 7 to emphasize the scaling feature.

\subsection{Scaling Analysis at Smaller Scales, Below $1000 \mathrm{~km}$}

In the above analysis, the whole basin as a unit was considered to calculate the spectra of WS and Hs. The results were nearly identical for different ocean basins. In order to better characterize the dynamical features of WS and Hs in different oceanic regions, datasets of finer scale are used to measure the power spectra. 

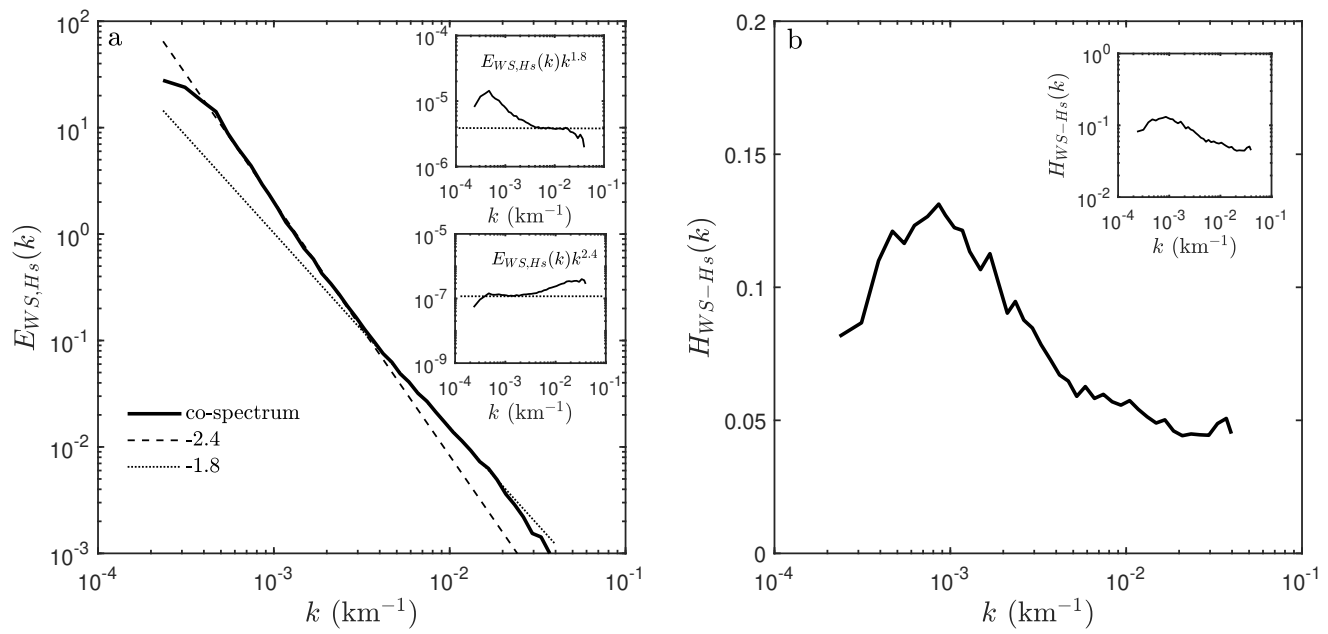

Figure 6. (a) Global averaged cross-spectrum of WS and Hs. The insets show the corresponding compensated spectra. (b) The coherence function between WS and Hs in the semi-log coordinate; the inset is in log-log plot.
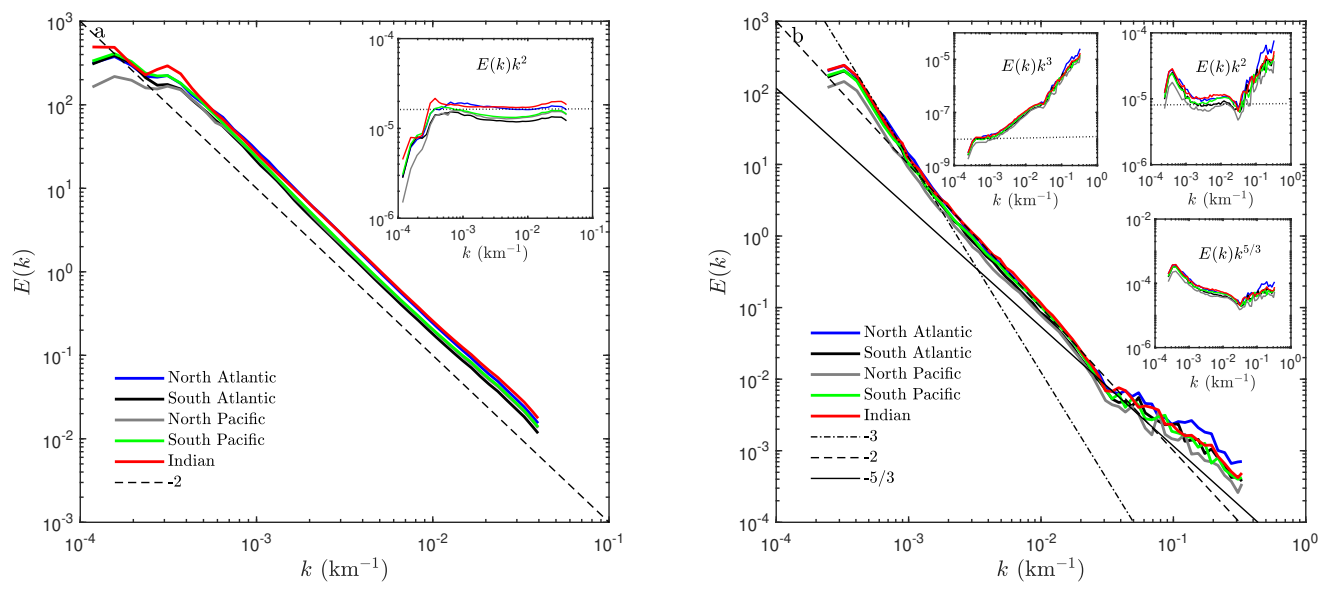

Figure 7. The ensemble averaged Fourier power spectra of the WS (a) and Hs (b) in different oceanic basins; the black dash dotted, dashed and thin lines are given as references. The insets show the corresponding compensated spectra. 

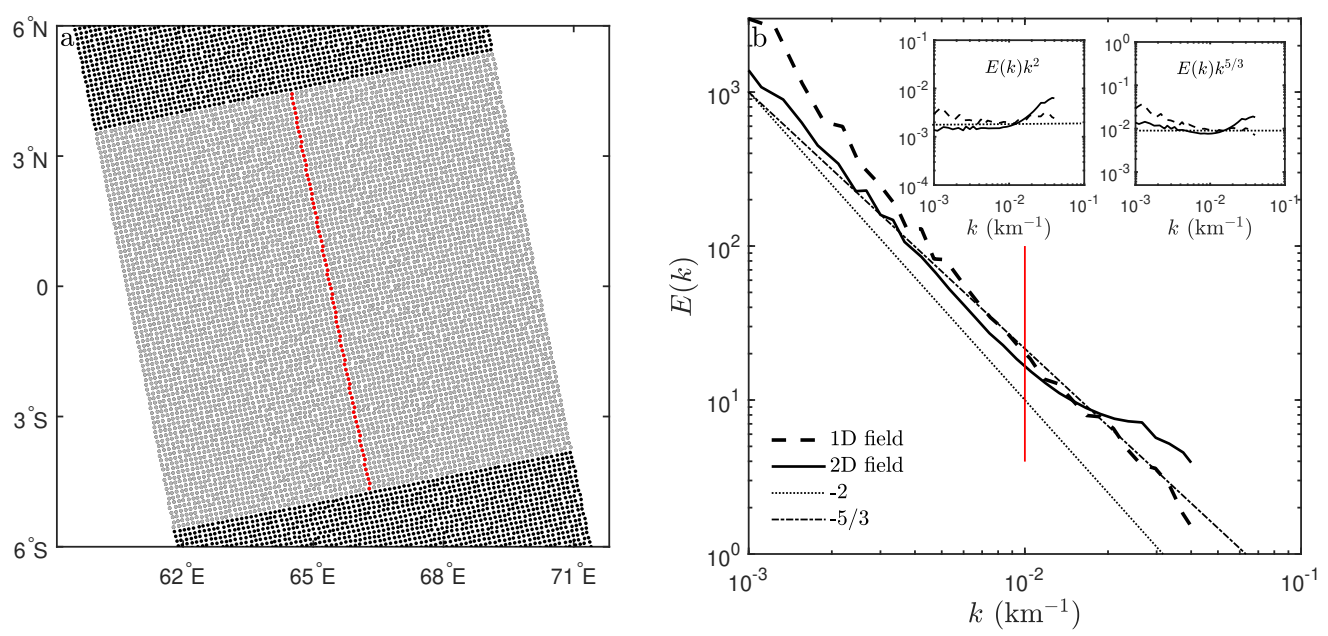

Figure 8. (a) An example of the selected $84 \times 842 \mathrm{D}$ domain of WS (gray dots); the line formed by red dots indicates the $1 \mathrm{D}$ field along the track of the satellite. (b) The global averaged Fourier power spectra measured from the 1D (dashed curve) and 2D (solid curve) WS subsets. The dotted line and dash-dot line are given as references with slopes equal to -2 and $-5 / 3$, respectively. The insets are the corresponding compensated spectra. The red line in (b) indicates the separation scale of the spectra, to emphasize the chosen scale, 100-1000 km.

For WS, the data were separated into $84 \times 84$ boxes for each orbit, each one being approximately a square area with a spatial scale of about $1000 \mathrm{~km} \times 1000 \mathrm{~km}$. Figure 8a gives an example of a 2D domain, in which the selected subset are shown as gray dots. The radially averaged Fourier power spectrum is then estimated by the aforementioned algorithm for the WS magnitude. For comparison, another approach to derive the scaling features for WS with the scales below $1000 \mathrm{~km}$ is to consider the 1D WS data along the satellite track (red dots in Figure 8a). The global averaged Fourier power spectra for the subsets of WS in 1D (along the track) and 2D (spatial zone) are illustrated in Figure 8b. Scaling features are found for both spectra on the scale range of 100-1000 km with a scaling exponent close to a value of 2 , see the compensated curves in the insets.

Concerning Hs, 700 along-track data points (which form a total of nearly $1000 \mathrm{~km}$ ) are selected as a whole to calculate the spectrum at smaller scales. In this way, the lowest wavenumber of spectra is around $1 \times 10^{-3} \mathrm{~km}^{-1}$. In order to increase the number of measurements to cover the global ocean, the selected data are overlapped $75 \%$ along the satellite track. Figure 9 illustrates the global averaged Fourier power spectrum for the Hs case. A scaling feature is distinguished in the range of $10-1000 \mathrm{~km}$, again with a scaling exponent close to a value of 2 .

The global distributions of scaling exponents for WS and Hs are derived by averaging the scaling exponents which are located on the same $2^{\circ} \times 2^{\circ}$ grid. This is done for the scale ranges chosen for each field (e.g., 100-1000 km for WS, and 10-1000 km for Hs). The results are illustrated in Figure 10 respectively (a) for WS and (b) for HS. The scaling exponents for WS and Hs both exhibit clear latitudinal dependence. Distinct boundaries which separate the scaling exponents whose values are larger or smaller than 1.8 are found around the horse latitudes (30 degrees north and south of the equator) for WS. Moreover, a small portion with large scaling exponents is found near the equatorial eastern Pacific (e.g., roughly $90^{\circ} \mathrm{W}$ to $160^{\circ} \mathrm{W}$ and $10^{\circ} \mathrm{S}$ to $10^{\circ} \mathrm{N}$ ), which coincidentally is in the same region as the famous Pacific equatorial dry zone (Hastenrath, 1999). For the nearby regions, such as the Intertropical Convergence Zone (ITCZ), equatorial Pacific Warm Pool (PWP), and 


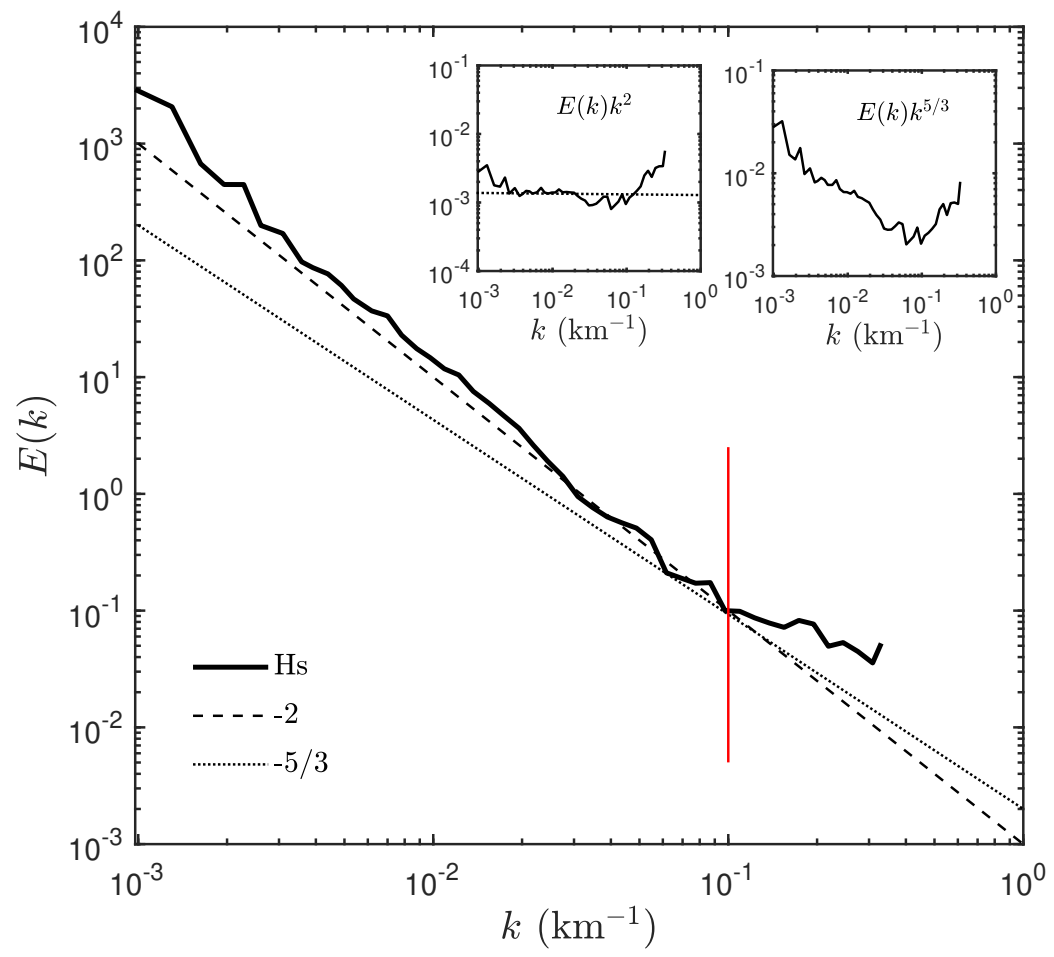

Figure 9. The global averaged Fourier power spectrum measured from the subsets of Hs; the dashed and dotted lines are given as references with slopes of -2 and $-5 / 3$. The insets are the corresponding compensated spectra. The red line indicates the separation scale of the spectrum to emphasize the chosen range, $10-1000 \mathrm{~km}$. 

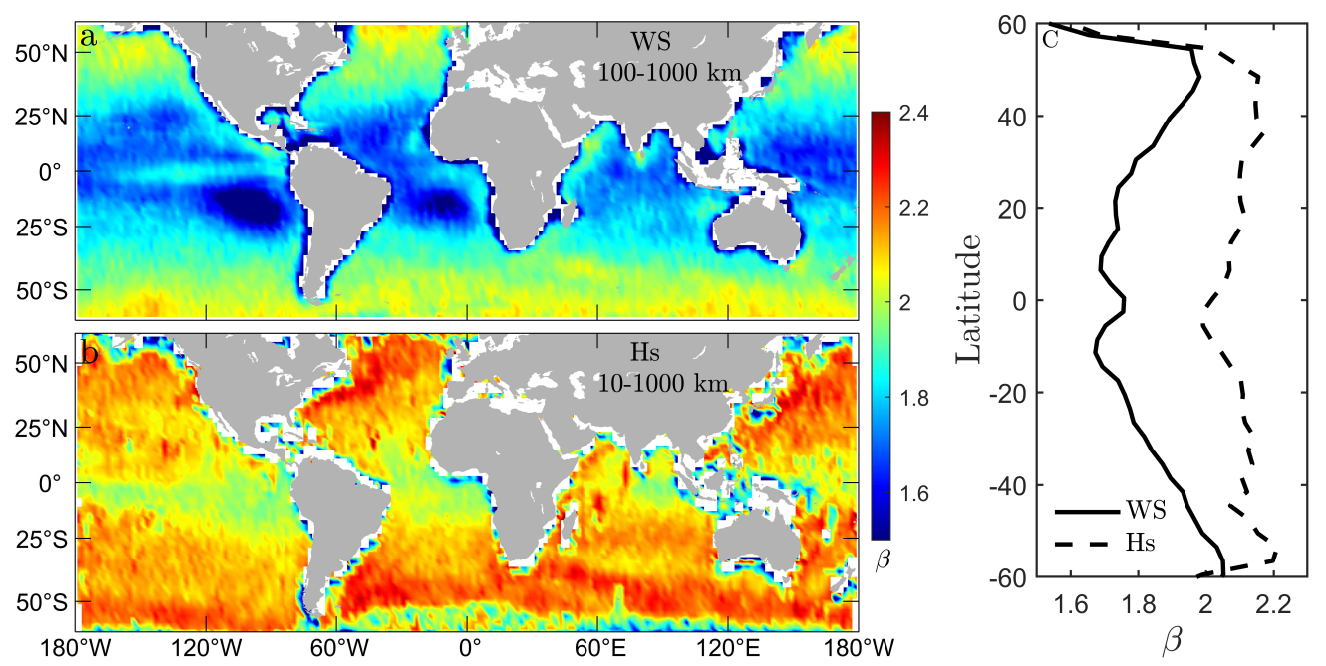

Figure 10. The global distribution of the scaling exponents $\beta$ measured from (a) WS (100-1000 $\mathrm{km}$ ) and (b) Hs data (10-1000 km). (c) The meridional variations of $\beta$ inside the Pacific Ocean. The solid and dashed curves indicate $\beta$ measured from WS and Hs, respectively.

South Atlantic Convergence Zone (SACZ), the scaling exponents for WS are close to $5 / 3$. For Hs, the scaling exponents are larger than the ones derived from WS for most regions, the meridional variations are relatively weak as compare to the WS case. Overall the global ocean, $\beta$ for Hs is larger than 2.1 except for the coastal areas. Again, relatively small $\beta$ is found in the tropical regions.

To quantify the meridional variation of $\beta$, a region inside the Pacific Ocean is chosen $\left(150^{\circ} \mathrm{E}\right.$ to $100^{\circ} \mathrm{W}, 60^{\circ} \mathrm{S}$ to $\left.60^{\circ} \mathrm{N}\right)$ to perform a meridional average of $\beta$. Figure $10 \mathrm{c}$ illustrates the measured meridional variations of $\beta$ for WS (solid curve) and Hs (dashed curve). The variation trends of $\beta$ for WS and Hs are similar, the maximums occur in mid-latitudes, with small values in tropical regions. Averaged $\beta$ derived from WS and Hs are mainly in between $1.6-2.2$ and $1.8-2.3$, respectively. A crest value can be found near the equator for WS case, which corresponds to the Pacific equatorial dry zone mentioned above.

Finally, we focus on the seasonal changes of these scaling parameters. For this, the difference $\Delta \beta=\beta_{\mathrm{s}}-\beta_{\mathrm{w}}$, where $\beta_{\mathrm{s}}$ is the summer scaling exponent, and $\beta_{\mathrm{w}}$ the winter one, is considered. The boreal summer is composed of the months of June, July, and August, and the boreal winter of the months of December, January, and February. The results are given in Figure 11. The seasonal variations of $\beta$ are weaker in mid-latitudes than those observed in low-latitudes, for both WS and Hs. The scaling exponents for WS are larger in winter than those in summer for most areas. While for Hs, the seasonal variations of $\beta$ are relatively complicated. For Northern Hemisphere, $\beta$ are larger in summer than those in winter for most regions; in the tropical area of Southern Hemisphere, $\beta$ for Hs is larger in winter, then for the regions further south than $25^{\circ} \mathrm{S}$, the seasonal differences are found to be vaguely.

\section{Discussion}

\subsection{Wind Data}

In this work, different scaling laws have been found for the wind velocity data set. First, the global averaged Fourier power spectrum for CFOSAT observed WS shows powerlaw features from 100 to $3000 \mathrm{~km}$ where $\beta$ varies from $5 / 3$ to 2.5 . Figure 5 a displaying the 

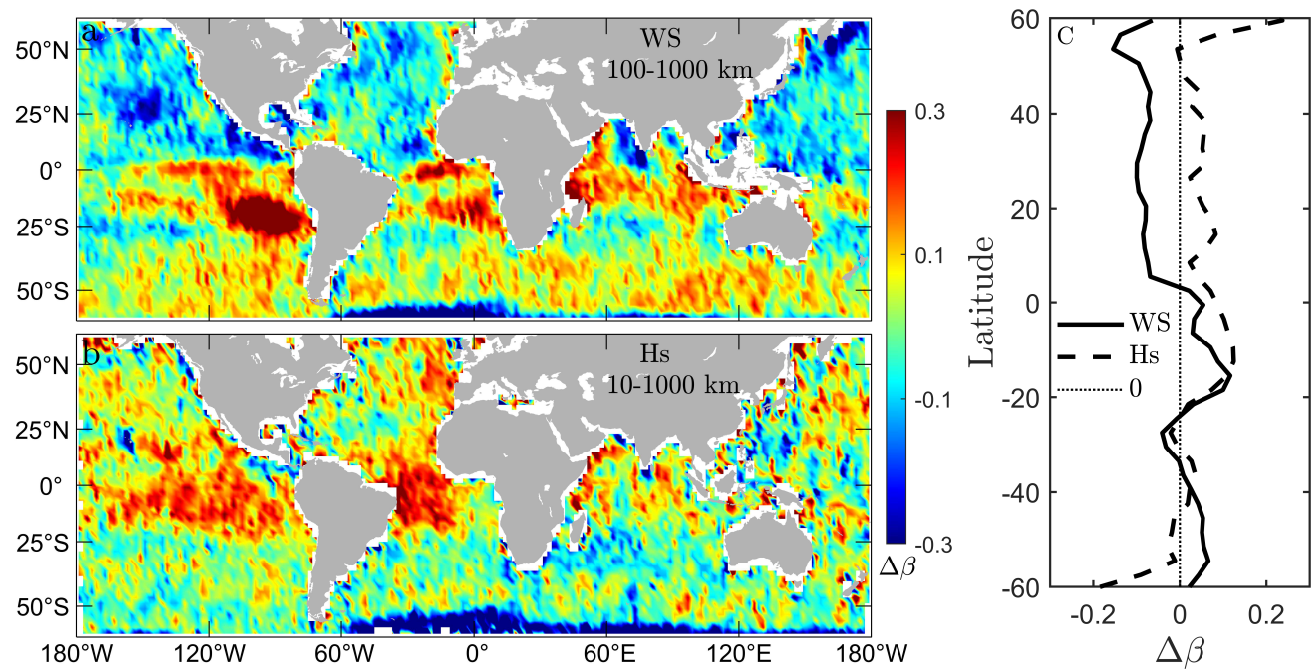

Figure 11. The global distribution of $\Delta \beta=\beta_{\mathrm{s}}-\beta_{\mathrm{w}}$, where $\beta_{\mathrm{s}}$ is the summer (June, July, and August) scaling exponent and $\beta_{\mathrm{w}}$ is the winter (December, January, and February) scaling exponent, for (a) WS in the wavelength band of 100-1000 km, and (b) Hs in the wavelength band of 10-1000 km. (c) The meridional variations of $\Delta \beta$ inside the Pacific Ocean. The solid and dashed curves indicate $\Delta \beta$ measured from WS and Hs, respectively.

global averaged Fourier spectra of zonal and meridional components of the wind field, shows that horizontally the wind field is roughly directionally isotropic, since the two curves follow the same laws as indicated by the insets. The scaling exponents are close to -2.5 for scales from 300 to $3000 \mathrm{~km}$, and close to $-5 / 3$ for smaller scales, from 30 to $300 \mathrm{~km}$, corresponding roughly to the mesoscale.

The fact that the meridional spectrum is slightly below the zonal spectrum is due to symmetry reasons. Indeed, this ratio is $3 / 4$ for $5 / 3$ isotropic turbulence, and it is a constant of the form $I(k)=2 /(1+\beta)$ for isotropic turbulence with spectral slope $\beta$ (Monin \& Yaglom, 1971). This ratio is displayed in Figure $5 \mathrm{~b}$ : it is close to $3 / 4$ for some range of scales for which there is no $5 / 3$ spectrum. Hence, the value found here is not a proof of horizontal symmetry, since for the scales for which an approximate $5 / 3$ slope is found (the larger wave numbers), the ratio is decreasing. This may be due to the fact that these spectra are not perfectly scaling. However, this ratio in log-log plot (inset of Figure $5 \mathrm{~b}$ ) still shows that there is no clear scaling difference between both spectra: the wind field data appear to be nearly directionally isotropic on the horizontal plane.

Note that previous studies have shown that the atmospheric movements could be anisotropic with different scaling exponents estimated along meridional, zonal, and vertical directions (Lovejoy et al., 2007, 2009; Pinel et al., 2014). For example, using the ECMWF reanalyses data, Lovejoy and Schertzer (2011) found horizontal anisotropy at 700 mbar, corresponding to the top of clouds. As aforementioned, the 10-meter wind field is in the marine-atmosphere boundary layer, where the horizontal directional isotropy might be restored due to the interaction with sea surface.

At the global scale, different regimes, climates, boundary conditions are mixed. The basin scale analysis is a way to avoid mixing signals from different zones, especially tropical zone which provides specific scaling properties. This is why the basin scale analyses show different results from the global analysis (Figure 4 versus Figure 7 ). Indeed at the basin scale 
there is a very neat power-law, from 25 to $2500 \mathrm{~km}$, with a scale ratio of 2 decades. Each basin shows the same universal property, which is quite remarkable.

To have results at finer scales, the analysis has been performed over shorter segments, over the range from 100 to $1000 \mathrm{~km}$. Globally the estimated $\beta$ displays patterns emphasizing the tropical regions, and latitude dependence. This latitude dependence could best be considered in the Pacific Ocean (Figure 10c), the differences in the value of $\beta$ are more marked at high latitudes. Such patterns show the climatic influences on wind scaling regimes.

Globally, no scaling range with $k^{-3}$ has been found here. It does not seem to sustain the enstrophy cascades corresponding to the quasigeostrophic theoretical framework. We find a $k^{-5 / 3}$ range, at mesoscales from 25 to $300 \mathrm{~km}$ (Figure $5 \mathrm{a}$ ), followed by an empirical fit close to the slope of -2.5 for larger scales, from 300 to $3000 \mathrm{~km}$, having no theoretical explanation. The more detailed analyses (Figure 10) show that the scaling exponents are not so universal and depend on the location. Strong patterns in this figure illustrate spatial variations of scaling features associated with different types of climate. Quite often in tropical regions, the exponents are close to $5 / 3$, whereas they are between 1.8 and 2.2 for the other areas (see also Figure 10c).

The -2 slopes that are found in the oceanic basins are in agreement with none of the theories discussed above. It was in fact already found in previous studies using satellite data. For instance, Freilich and Chelton (1986) examined the Seasat-A satellite scatterometer observed wind vector data over the Pacific Ocean, and found an energy spectrum proportional to $k^{-2}$ in the range of 200 to $2200 \mathrm{~km}$. Pinardi and Milliff (2004) found also a $k^{-2}$ slope for the spectrum of the Mediterranean surface winds from QuikSCAT for spatial scales between $200 \mathrm{~km}$ and $1000 \mathrm{~km}$. Chelton et al. (2006) studied the wavenumber spectra of the QuikSCAT zonal and meridional wind components and the wind speed in the North Pacific. The results shown that the dependence on wavenumber $k$ for these three variables are all approximately $k^{-2}$ for scales below $1000 \mathrm{~km}$.

We may compare our results with other previous studies. Wikle et al. (1999) considered wind data from three different sources (e.g., reanalysis wind data from National Centers for Environmental Prediction, satellite-based ERS-1 scatterometer observed wind, and wind from high-resolution aircraft observations). They found that the combined spectra from these data demonstrate a power-law relation over the range 1-1000 km, with a best-fit slope close to the value of $-5 / 3$, and the energy spectra for subsets of the data support spectral slopes of $-5 / 3$ and -2 . Patoux and Brown (2001) investigated the spectra of QuikSCAT observed wind vectors. They fitted the spectra on the range of $100-1000 \mathrm{~km}$ to derive the scaling exponents. The values of measured $\beta$ were roughly between $5 / 3$ and 3 , with wide variations from 1.8 to 2.6. Then the spatial and seasonal variations of the spectra slopes were examined. They found that the energy spectra were steeper in midlatitudes, and all the more so in winter, also with steeper slopes in the tropics in the presence of convection. They concluded that when convection is enhanced, the energy level is raised at all scales. Consequently, the energy spectrum is steeper. The seasonal variations correspond to enhanced baroclinic activities in winter in the midlatitudes. According to our results, in the strong convection regions, e.g., ITCZ, PWP, and SACZ, the measured $\beta$ in 100-1000 km scales is close to $5 / 3$. The corresponding spectra are flatter than the ones in nearby regions, i.e., equatorial dry zone with relatively steady atmospheric movements. Xu et al. (2011) performed a spectral analysis of QuikSCAT winds over the global ocean, and found that $\beta$ possesses spatial variability in the scale range of $1000-3000 \mathrm{~km}$, with values varying from 1.6 to 2.9. The slopes of spectra were observed to become steeper toward high-latitudes in the Pacific and in the South Atlantic. Besides, the spectra are steeper in winter than those in summer for most regions of the Northern Hemisphere midlatitude. This was also explained by the baroclinic argument. Furthermore, the seasonal differences which are observed in our analysis might also be related to the temporal variations of the baroclinic instability strength. 
Globally, the analysis for WS subsets found in the present study is relatively consistent with the results reported by Patoux and Brown (2001) and Xu et al. (2011). With the same fitting range set as Patoux and Brown (2001), we found $\beta$ for WS subsets various from 1.6 to 2.2 for most regions, and $\beta$ are larger in midlatitudes than those in tropics. The regions in same latitudes but with strong convection show $\beta$ close to $5 / 3$.

Let us mention some limitations or specific properties of CFOSAT data used in this study. The wind speed retrieved by the SCAT sensor is typically treated as 10 meters above the sea surface. This height is close to the bottom of the marine atmospheric boundary layer, which is often in the order of $\mathcal{O}(1) \mathrm{km}$ (Lang et al., 2018). The 10-meter wind is thus strongly influenced by the bottom conditions, e.g., the effective roughness/wave height, temperature profiles, the variation of boundary layer, to name a few. Hence, it is not purely large-scale turbulence and boundary conditions may have influence on the statistics. For the same reason, one cannot fully compare the results from this sensor to the one of the altitude measurements using the commercial aircraft, such as the one obtained by Nastrom and Gage (1985) or other similar measurements done at the height $\mathcal{O}(10) \mathrm{km}$.

\subsection{Wave Data}

From global analysis, features for $\mathrm{Hs}$ are found in the ranges of 10 to $1000 \mathrm{~km}$ and 1000 to $3000 \mathrm{~km}$ with $\beta$ close to 2 and 3, respectively. Some heave and roll features that occur in the spectrum range below $50 \mathrm{~km}$ may be the signature of energy injections induced by some energetic submesoscale processes, such as eddies and fronts or other interactions between surface currents, closely related to the upper ocean dynamics, thermodynamics, and biogeochemistry. At the basin scale, spectra for $\mathrm{Hs}$ in different oceanic basins are identical in the spatial ranges of 50 to $2000 \mathrm{~km}$ (Figure 7). As indicated above, there is no theory to explain such results. We may only compare these findings with previous published works. Similar studies estimating the spectra for satellite collected Hs were performed by Monaldo (1988, 1990), where 100 Geosat radar altimeter sampled Hs trajectories around the world were used to calculate the energy spectra. The scaling exponents were found to be around 1.4 in the spatial scale from several kilometers to about $50 \mathrm{~km}$. Similar scaling features are observed in our study at small scales (Figure 4 and Figure 7b) where both global and basin scale averaged spectra are shown. For the scales less than $50 \mathrm{~km}$, the scaling exponents are slightly smaller than $3 / 5$. Tournadre (1993) estimated the energy spectra with 583 and 689 Geosat observed Hs trajectories. The corresponding scaling exponents were derived in the range 14 to $1400 \mathrm{~km}$ with the values equal to 1.39 and 1.21, in the North Sea and equatorial Atlantic respectively. Spatial differences are found, and values of $\beta$ are larger in midlatitudes than those in tropics, which is similar to what we found of the spatial distribution of $\beta$ in the scale of 10-1000 km, while in our results the slopes are steeper.

The parameter Hs is a measure of the local roughness, proportional to the small-scale variance of the wave field. This quantity shows a scale-dependent variability, with long-range correlations as revealed by the scaling regimes which have been found. Such properties of Hs are certainly inherited from the surface wave height field, for which several scaling theories have been proposed in the literature, as was discussed in a previous section.

\subsection{Coupling between Wind and Waves}

Wind and waves are closely related to each other, and the relationship between WS and Hs has been studied for a long time (Kinsman, 1965; Carter, 1982; Andreas \& Wang, 2007; Khandekar, 2013). Due to the limitation of data available, the dynamic features between WS and Hs in the spatial domain are rarely reported in literature. Bhandari and Shaeb (2014) examined the spatial coherence of WS and Hs over the Arabian Sea with autocorrelation functions. They found that the spatial coherence scales are of the order of 100 to $500 \mathrm{~km}$. The Hs and WS data they used were all provided by Poseidon-2 Radar Altimeter. The WS could have been retrieved from altimeter, but the accuracy is relatively poor as 
compared to the ones got from scattermeter (Monaldo, 1988; Hwang et al., 1998; Ribal \& Young, 2019). In our study, based on the SCAT observed WS and SWIM collected Hs, the co-spectrum of WS and Hs was derived at a global scale. Scaling features were found in the spatial ranges from 30 to $250 \mathrm{~km}$ and 250 to $2000 \mathrm{~km}$ with scaling exponents equal to 1.8 and 2.4, respectively. Further, the coherence function also illustrated scale dependent features. These results confirmed the existence of spatially coupled dynamical links between WS and Hs, and the fact that the mechanisms of these links are related to the spatial scales. However, the fundamental rules of the interactions between WS and Hs are still unclear and will be the topic of further works.

\section{Conclusion}

Simultaneously observed wind and wave data by CFOSAT have been used to determine the multiscale features of WS and Hs. Before processing the data, quality controls have been carefully performed and outliers were removed, as shown by the pdfs of the two main variables considered here. The spatial and temporal variations of the corresponding scaling exponents have been examined. Power-law features were found in the global averaged Fourier power spectra of WS and Hs. The scaling exponents $\beta$ for Hs are close to 3 , and 2 in the range of $1000-3000 \mathrm{~km}$ and $10-1000 \mathrm{~km}$, respectively. For WS, power-law features are found from 100 to $1000 \mathrm{~km}$ with $\beta$ between $5 / 3$ and 2 , with little evidence of $\beta$ close to 3 for larger scales. The global averaged co-spectrum for WS and Hs also showed power-law features from $30 \mathrm{~km}$ to $250 \mathrm{~km}$ and $250 \mathrm{~km}$ to $2000 \mathrm{~km}$ with scaling exponents equal to 1.8 and 2.4, respectively. The values of the coherence function lay between 0.04 and 0.13 in the whole spatial ranges, and the maximum occurs around at $1200 \mathrm{~km}$.

Our results showed that within each oceanic basin, the averaged Fourier power spectra for WS and Hs have nearly identical shapes and scaling features from $50 \mathrm{~km}$ to $3000 \mathrm{~km}$, while for the scales below $50 \mathrm{~km}$, the spectra for $\mathrm{Hs}$ show more roughness as compared to those at larger scales. This may indicate that complex submesoscale processes are dominant at these scales.

A finer examination of the global distribution of $\beta$ showed that the scaling exponents for WS and Hs are both meridional dependent with large values occurring in middle and high latitudes and small values in the tropics. For WS, the scaling exponents are smaller inside the convergence zones than those in the relatively steady equatorial dry zone. The scaling exponents in convective regions are close to $5 / 3$. We also considered the temporal evolution of scaling exponents. Their seasonal variations derived from WS data showed small scaling exponents in summer and large ones in winter for most regions. This result is likely related to the strength of baroclinic disturbances in different seasons. While for Hs case, $\beta$ are found larger in summer than those in winter in the Northern Hemisphere; in the tropical area of Southern Hemisphere, large $\beta$ is found in winter, as for the regions further south than $25^{\circ} \mathrm{S}$, the seasonal variations are unclear.

Globally these results show that some scaling properties may be obtained when considering all the data together, some universal properties are found when considering the averages in some subsamples, such as the basin scale study, especially for the wind field when a very clear $k^{-2}$ range was found over 2 decades in scale. The variability of the field considered, and hence their spectral properties, vary in time and in space and some adequate spatial or temporal domain must be chosen to find evolution, universality, patterns and identify hidden processes. This is what has been done in this work, either by looking at the basin scale, the local spatial patterns, the latitude averages in one ocean, or the seasonal changes in some spatial patterns.

In this work, some basic scaling features of WS and Hs collected by CFOSAT have been derived by spectral analysis. For further works, we plan to characterize also the intermittency in these scaling ranges, by considering structure functions, joint structure 
functions and also Hilbert spectral analysis (HSA) of higher orders, which are shown not to be strongly impacted by energetic forcing (Huang et al., 2008, 2011; Schmitt \& Huang, 2016). Also, the information provided by one satellite with a single scaling analysis approach is not enough to further diagnose the multiscale dynamical features of WS and Hs. Studies of WS and Hs data from other satellites or in-situ observations with other compensatory scaling analysis methods are needed. Finally, it is planned also to focus more on the dynamical relationships between WS and Hs.

\section{Acknowledgments}

The suggestions by two reviewers, that lead to substantial improvements, are acknowledged. This work was supported by the CNES (French space agency). It is based on observations with SCAT and SWIM embarked on CFOSAT China-France satellite. This work was supported by the National Natural Science Foundation of China (NSFC) (Grant Nos. 91958203 and 11732010). This research has been performed in the frame of the MultiW2 project, funded by the CNES/TOSCA program. Funding of YG's Cotutella doctoral research project by Région Hauts-de-France and Xiamen University is acknowledged. The CNRS-NSFC project TURBLOOMS (Turbulence and marine algal blooms : use of satellite remote detection; 2019-2021) is acknowledged for partial financial support. YX is partially supported by State Key Laboratory of Ocean Engineering (Shanghai Jiao Tong University) (Grant No. 1910). The CFOSAT data used in this study can be freely accessed at https://www.aviso.altimetry.fr. The Matlab and Python codes are freely available at https://github.com/lanlankai. The authors are grateful to the team of China France Oceanography Satellite. 


\section{Appendix A CFOSAT Orbits}

In the main text, we mentioned that the CFOSAT provides a global coverage every roughly 3 days for both the wind field and waves. The CFOSAT orbit is fixed: it cannot provide a 24 -hour observation for the same region, see Figure A1.
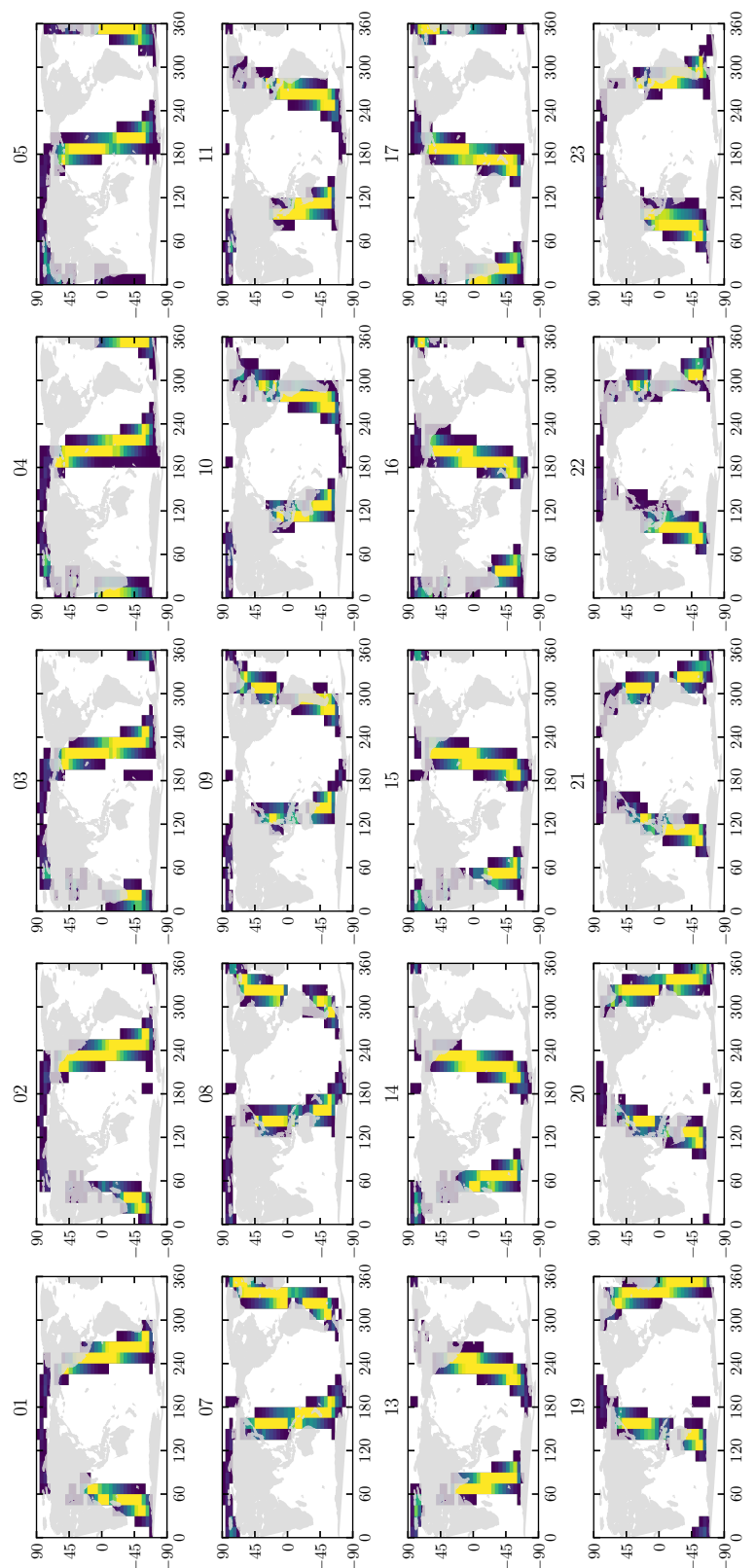

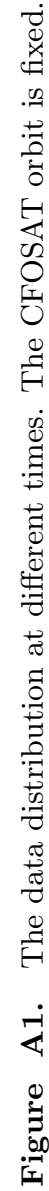
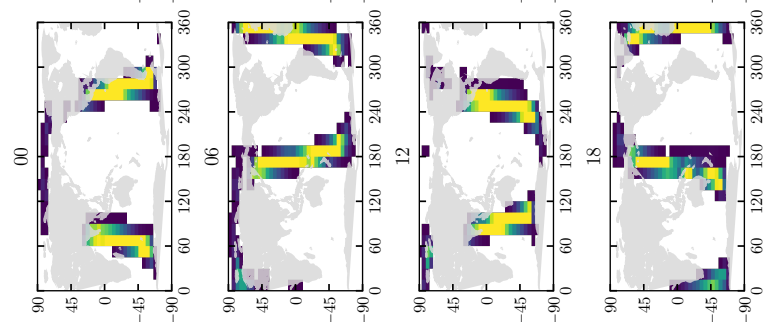


\section{Appendix B Effect of outliers on $\beta$}

Here, we present the effect of outliers on the slope of spectra, by subtracting the measured $\beta$ with raw data from the ones estimated with data after quality control (QC). The results are illustrated in Figure B1. There are clear spatial variations for both WS and Hs. For WS, the existence of abnormal values leads to flatter spectra in tropical areas (except in ITCZ). While for the other higher latitudes, the outliers make the spectra relatively steep as compared to the ones measured with the data after QC. The differences are all in between -0.2 to 0.2 . For Hs, the influence of outliers is mainly found along the coastal and equatorial regions, where the slopes of the spectra are greatly reduced. For the other regions (open oceans), SWIM performs well in Hs observation, since there are relatively small estimation errors. Thus, the differences of $\beta$ in the open oceans are close to 0 for most regions.

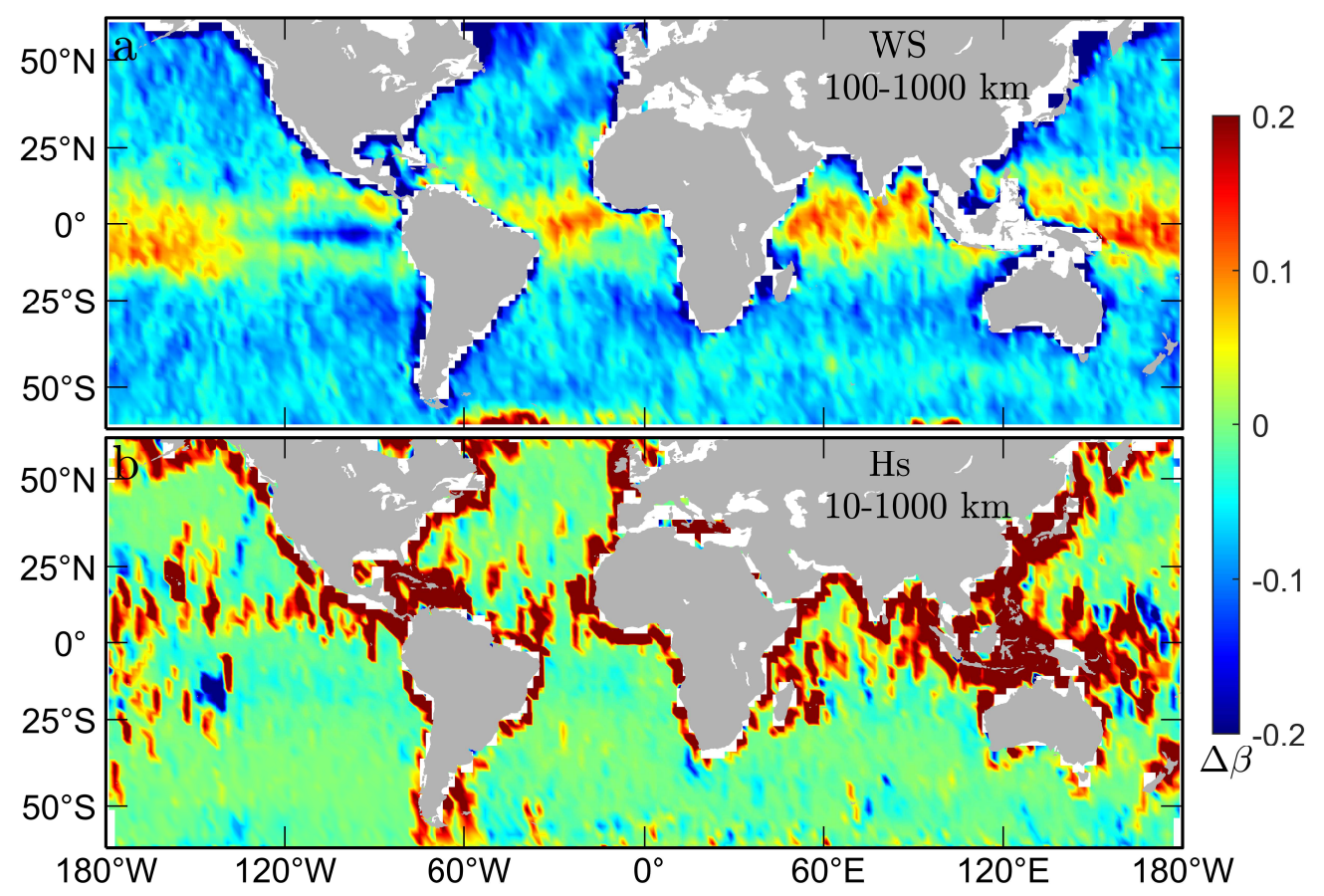

Figure B1. The global distribution of $\Delta \beta=\beta_{Q}-\beta_{R}$, where $\beta_{Q}$ and $\beta_{R}$ are the scaling exponents estimated with data after QC and raw data, respectively. For (a) WS in the wavelength band of $100-1000 \mathrm{~km}$, and (b) Hs in the wavelength band of 10-1000 km. 


\section{Appendix C Code for Quality Control}

Matlab version codes to perform the quality control is provided below, see Figure C1. Both Matlab and Python codes are also freely available at: https://github.com/lanlankai.

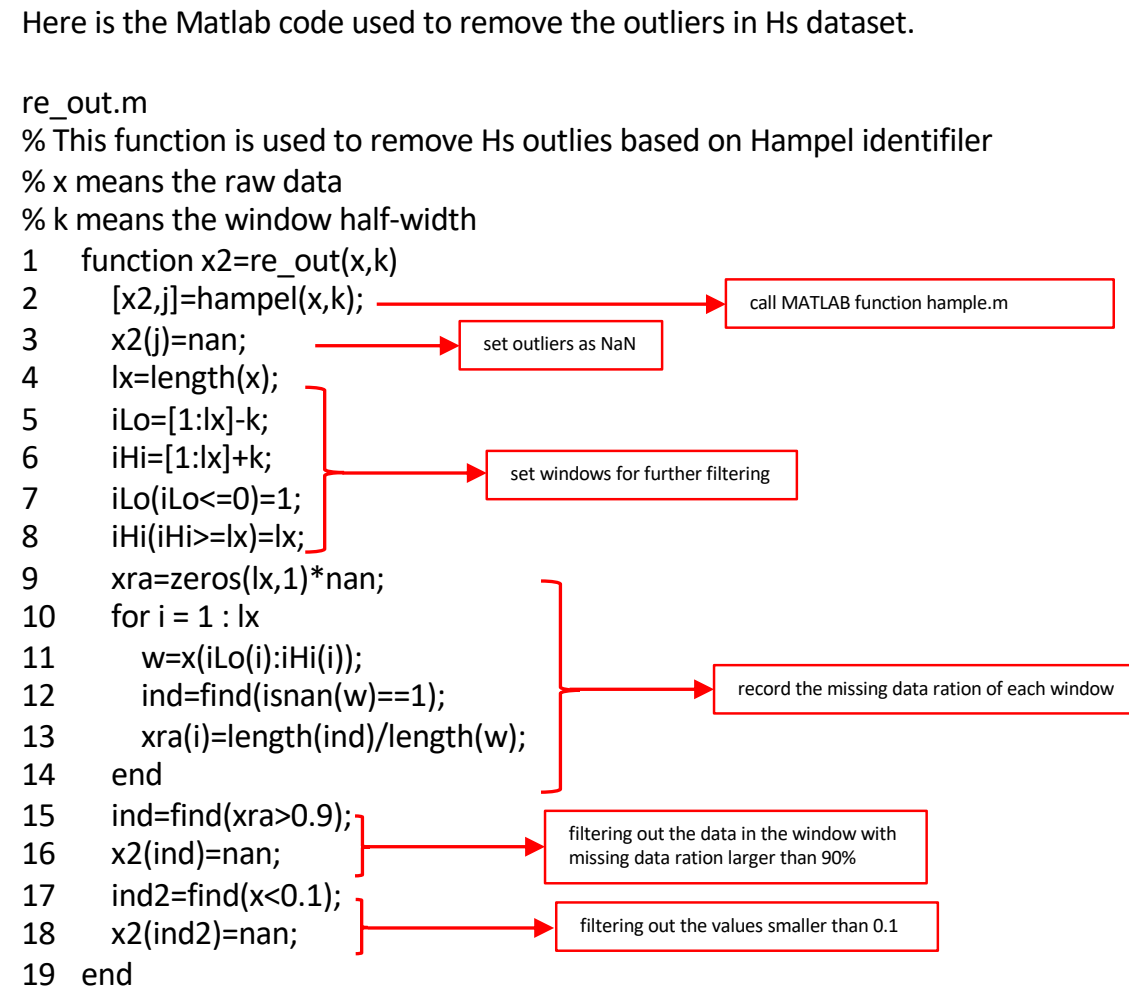

Figure C1. A Matlab code to remove the outliers. 


\section{Appendix D Code for the Wiener-Khinchine Theorem Preserved Fourier Power Spectrum}

Matlab version codes to estimate the Fourier power spectrum via the Wiener-Khinchine Theorem (WKT) for both 1D and 2D cases are provided below, see Figure D1, D2 and D3.

${ }_{647}$ Both Matlab and Python codes are also freely available at: https://github.com/lanlankai. 


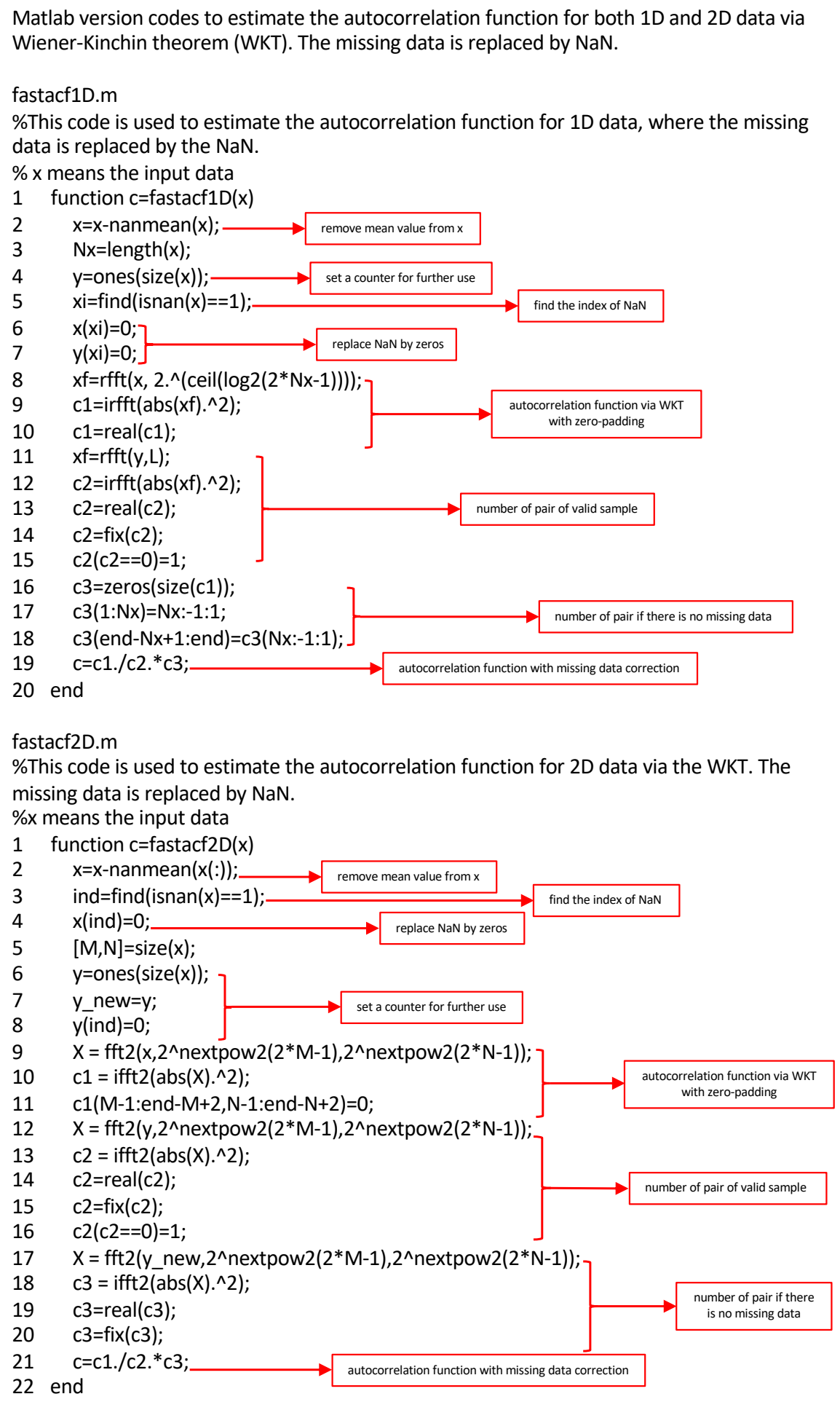

Figure D1. A Matlab code to estimate the auto-correlation function in both 1D and 2D with the missing data correction.

\section{References}

Andreas, E. L., \& Wang, S. (2007). Predicting significant wave height off the northeast 


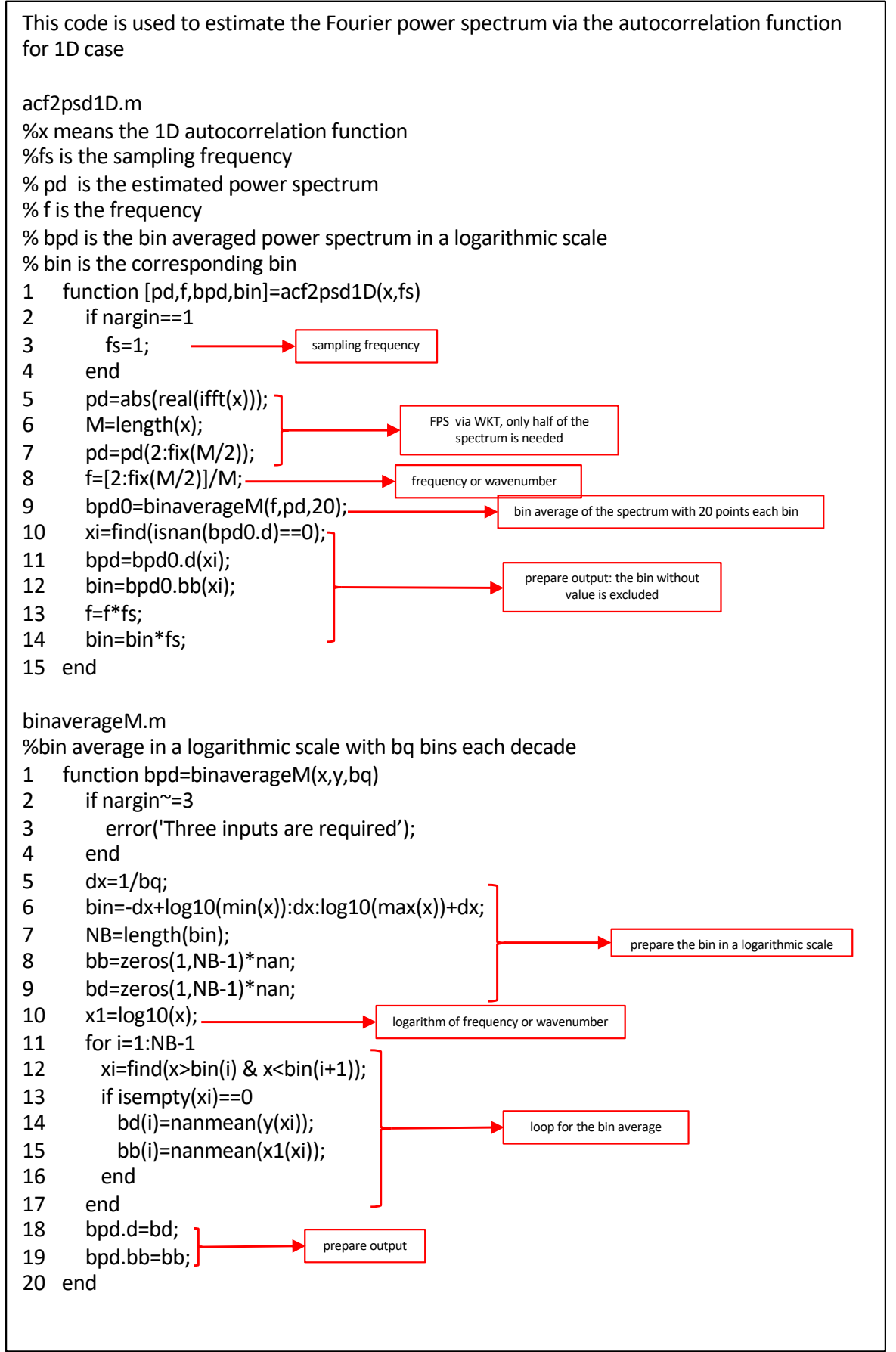

Figure D2. A Matlab code to estimate the 1D Fourier power spectrum and to perform a bin average in a log-scale.

coast of the United States. Ocean Engineering, 34(8-9), 1328-1335.

Babanin, A. (2006). On a wave-induced turbulence and a wave-mixed upper ocean layer. Geophysical Research Letters, 33(20), L20605.

Belcher, S., \& Vassilicos, J. (1997). Breaking waves and the equilibrium range of wind-wave. Journal of Fluid Mechanics, 342, 377-401.

Bhandari, S., \& Shaeb, K. H. B. (2014). Spatial Coherence of winds and waves over the 


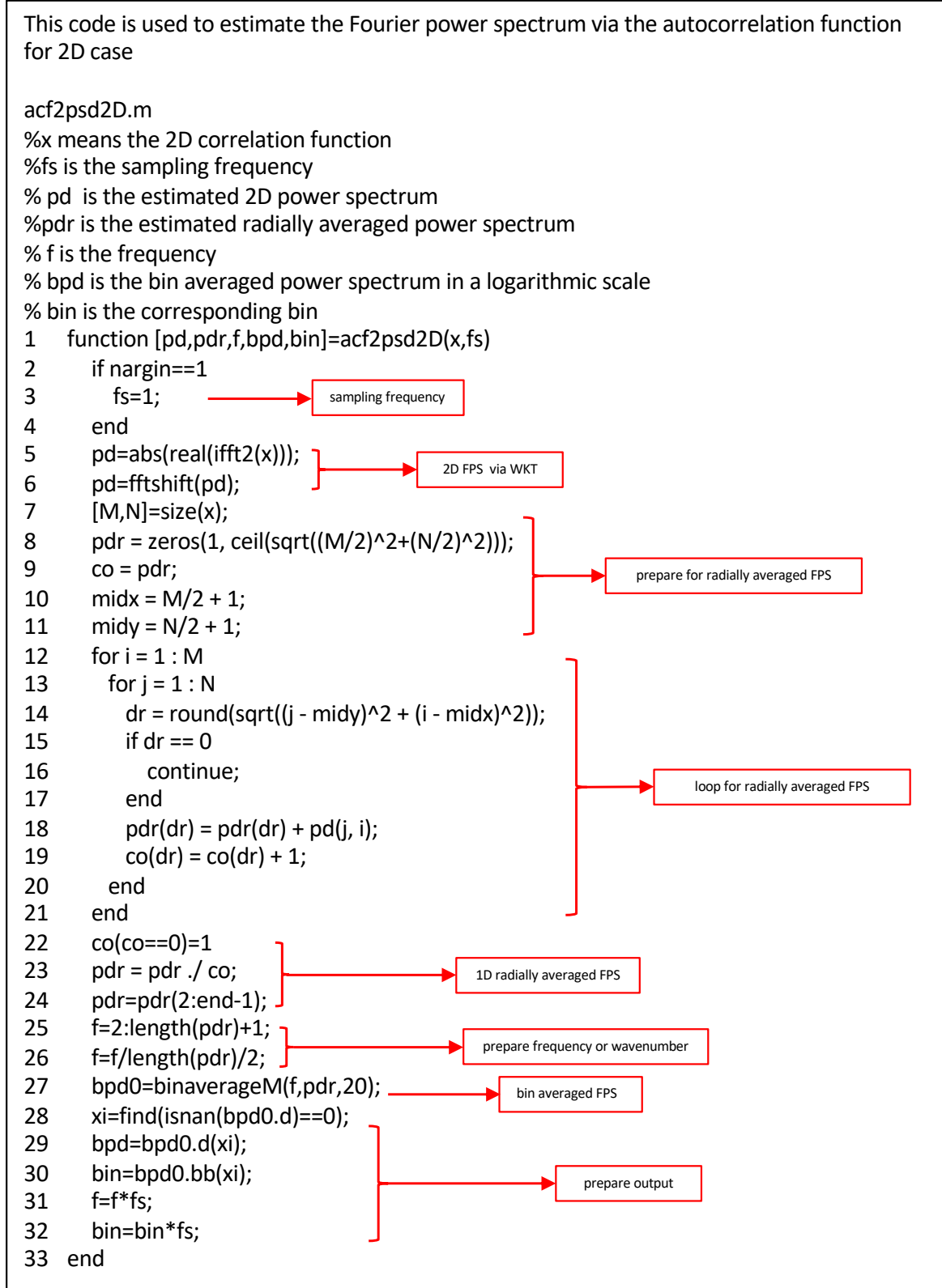

Figure D3. A Matlab code to estimate the 2D Fourier power spectrum and to perform a radially average.

Arabian Sea and Bay of Bengal and their evolution during SW Monsoon: a novel application of along-track Radar Altimeter measurements from space. International Archives of the Photogrammetry, Remote Sensing 6 Spatial Information Sciences, XL-8, 1027-1033.

Bigg, G., Jickells, T., Liss, P., \& Osborn, T. (2003). The role of the oceans in climate. International Journal of Climatology: A Journal of the Royal Meteorological Society, $23(10), 1127-1159$.

Bolgiano, R. (1959). Turbulent spectra in a stably stratified atmosphere. Journal of Geoscience Research, 64, 2226-29.

Brown, G. (1977). The average impulse response of a rough surface and its applications. 
IEEE Transactions on Antennas and Propagation, 25(1), 67-74.

Calif, R., \& Schmitt, F. G. (2012). Modeling of atmospheric wind speed sequence using a lognormal continuous stochastic equation. Journal of Wind Engineering and Industrial Aerodynamics, 109, 1-8.

Carter, D. (1982). Prediction of wave height and period for a constant wind velocity using the JONSWAP results. Ocean Engineering, 9(1), 17-33.

Charney, J. G. (1971). Geostrophic turbulence. Journal of the Atmospheric Sciences, 28(6), 1087-1095.

Chelton, D. B., Freilich, M. H., Sienkiewicz, J. M., \& Von Ahn, J. M. (2006). On the use of QuikSCAT scatterometer measurements of surface winds for marine weather prediction. Monthly Weather Review, 134(8), 2055-2071.

Danilov, S. D., \& Gurarie, D. (2000). Quasi-two-dimensional turbulence. Physics-Uspekhi, $43(9), 863$.

Davies, L., \& Gather, U. (1993). The identification of multiple outliers. Journal of the American Statistical Association, 88(423), 782-792.

Egolf, P. W., \& Hutter, K. (2020). Nonlinear, nonlocal and fractional turbulence: Alternative recipes for the modeling of turbulence. Springer Nature.

Formenti, D. (1999). What is the coherence function and how can it be used to find measurement and test setup problems? Sound and Vibration, 33(12), 2-3.

Freilich, M., \& Chelton, D. (1986). Wavenumber spectra of Pacific winds measured by the Seasat scatterometer. Journal of Physical Oceanography, 16(4), 741-757.

Frisch, U. (1995). Turbulence: the legacy of AN Kolmogorov. Cambridge University Press.

Hanley, K. E., Belcher, S. E., \& Sullivan, P. P. (2010). A global climatology of wind-wave interaction. Journal of Physical Oceanography, 40(6), 1263-1282.

Hastenrath, S. (1999). Dynamics of the Pacific equatorial dry zone. Meteorology and Atmospheric Physics, 71 (3), 243-254.

Hauser, D., Tison, C., Amiot, T., Delaye, L., Mouche, A., Guitton, G., ... Castillan, P. (2016). CFOSAT: A new Chinese-French satellite for joint observations of ocean wind vector and directional spectra of ocean waves. In R. J. Frouin, S. C. Shenoi, \& K. H. Rao (Eds.), Remote sensing of the oceans and inland waters: Techniques, applications, and challenges (Vol. 9878, pp. 117-136). SPIE.

Hauser, D., Tourain, C., Hermozo, L., Alraddawi, D., Aouf, L., Chapron, B., ... Tran, N. (2020). New observations from the SWIM radar on-board CFOSAT: instrument validation and ocean wave measurement assessment. IEEE Transactions on Geoscience and Remote Sensing.

Huang, Y., Schmitt, F. G., Hermand, J.-P., Gagne, Y., Lu, Z., \& Liu, Y. (2011). Arbitraryorder Hilbert spectral analysis for time series possessing scaling statistics: Comparison study with detrended fluctuation analysis and wavelet leaders. Physical Review E, 84(1), 016208.

Huang, Y., Schmitt, F. G., Lu, Z., \& Liu, Y. (2008). Scaling analysis of time series using empirical mode decomposition and Hilbert spectral analysis. Traitement du Signal, 25(6), 481-492.

Hwang, P. A., Teague, W. J., Jacobs, G. A., \& Wang, D. W. (1998). A statistical comparison of wind speed, wave height, and wave period derived from satellite altimeters and ocean buoys in the Gulf of Mexico region. Journal of Geophysical Research: Oceans, 103(C5), 10451-10468.

Khandekar, M. L. (2013). Operational analysis and prediction of ocean wind waves (Vol. 33). Springer Science \& Business Media.

Kinsman, B. (1965). Wind waves: their generation and propagation on the ocean surface. Englewood Cliffs, N.J. Prentice-Hall.

Kirchner, J. W. (2005). Aliasing in $1 / f^{\alpha}$ noise spectra: Origins, consequences, and remedies. Physical Review E, $71(6), 066110$.

Kolmogorov, A. (1941). Energy dissipation in locally isotropic turbulence. Doklady Akademii Nauk SSSR, 32, 19-21. 
Kraichnan, R. H. (1967). Inertial ranges in two-dimensional turbulence. Physics of Fluids, 10(7), 1417-1423.

Kunz, G. J., de Leeuw, G., Becker, E., \& O'Dowd, C. D. (2002). Lidar observations of atmospheric boundary layer structure and sea spray aerosol plumes generation and transport at Mace Head, Ireland (PARFORCE experiment). Journal of Geophysical Research: Atmospheres, 107(D19), PAR-11.

Lang, F., Huang, Y., Siems, S., \& Manton, M. (2018). Characteristics of the marine atmospheric boundary layer over the southern ocean in response to the synoptic forcing. Journal of Geophysical Research: Atmospheres, 123, 7799-7820.

Li, X., Xu, Y., Liu, B., Lin, W., He, Y., \& Liu, J. (2021). Validation and calibration of Nadir SWH products from CFOSAT and HY-2B with satellites and in-situ observations. Journal of Geophysical Research: Oceans, 126, e2020JC016689.

Lin, W., \& Dong, X. (2011). Design and optimization of a Ku-band rotating, range-gated fanbeam scatterometer. International Journal of Remote Sensing, 32(8), 2151-2171.

Lin, W., Dong, X., Portabella, M., Lang, S., He, Y., Yun, R., .. Liu, J. (2018). A perspective on the performance of the CFOSAT rotating fan-beam scatterometer. IEEE Transactions on Geoscience and Remote Sensing, 57(2), 627-639.

Liu, J., Lin, W., Dong, X., Lang, S., Yun, R., Zhu, D., .. others (2020). First results from the rotating fan beam scatterometer onboard cfosat. IEEE Transactions on Geoscience and Remote Sensing, 58(12), 8793-8806.

Lovejoy, S., \& Schertzer, D. (2011). Space-time cascades and the scaling of ECMWF reanalyses: Fluxes and fields. Journal of Geophysical Research: Atmospheres, 116(D14).

Lovejoy, S., \& Schertzer, D. (2013). The weather and climate: emergent laws and multifractal cascades. Cambridge University Press.

Lovejoy, S., Tuck, A., Hovde, S., \& Schertzer, D. (2007). Is isotropic turbulence relevant in the atmosphere? Geophysical Research Letters, 34(15).

Lovejoy, S., Tuck, A., Schertzer, D., \& Hovde, S. (2009). Reinterpreting aircraft measurements in anisotropic scaling turbulence. Atmospheric Chemistry \& Physics, 9(14), $5007-5025$.

Monaldo, F. (1988). Expected differences between buoy and radar altimeter estimates of wind speed and significant wave height and their implications on buoy-altimeter comparisons. Journal of Geophysical Research: Oceans, 93(C3), 2285-2302.

Monaldo, F. (1990). Corrected spectra of wind speed and significant wave height. Journal of Geophysical Research: Oceans, 95(C3), 3399-3402.

Monin, A., \& Yaglom, A. M. (1971). Statistical fluid mechanics, vols. 1 and 2 (Vol. 1975). MIT Press, Cambridge, MA.

Nastrom, G., \& Gage, K. S. (1985). A climatology of atmospheric wavenumber spectra of wind and temperature observed by commercial aircraft. Journal of the Atmospheric Sciences, 42(9), 950-960.

Obukhov, A. (1941). Spectral energy distribution in a turbulent flow. Izvestiya Akademii Nauk SSSR, Seriya Geografii i Geofiziki, 5, 453-466.

Obukhov, A. (1959). On influence of buoyancy forces on the structure of temperature field in a turbulent flow. Doklady Akademii Nauk SSSR, 125, 1246.

Patoux, J., \& Brown, R. A. (2001). Spectral analysis of QuikSCAT surface winds and twodimensional turbulence. Journal of Geophysical Research: Atmospheres, 106(D20), 23995-24005.

Pearson, R. K. (2002). Outliers in process modeling and identification. IEEE Transactions on Control Systems Technology, 10(1), 55-63.

Pearson, R. K., Neuvo, Y., Astola, J., \& Gabbouj, M. (2015). The class of generalized Hampel filters. In 2015 23rd European Signal Processing Conference (EUSIPCO) (pp. 2501-2505).

Pearson, R. K., Neuvo, Y., Astola, J., \& Gabbouj, M. (2016). Generalized Hampel filters. EURASIP Journal on Advances in Signal Processing, 2016(1), 1-18.

Phillips, O. (1985a). The equilibrium range in the spectrum of wind-generated waves. Journal of Fluid Mechanics, 4, 426-434. 
Phillips, O. (1985b). Spectral and statistical properties of the equilibrium range in windgenerated gravity waves. Journal of Fluid Mechanics, 156, 505-531.

Pinardi, N., \& Milliff, R. F. (2004). Comparing the kinetic energy vs. wavenumber in surface wind fields from ecmwf analyses and the nasa quikscat scatterometer (Tech. Rep.). Istituto Nazionale di Geofisica e Vulcanologias.

Pinel, J., \& Lovejoy, S. (2014). Atmospheric waves as scaling, turbulent phenomena. Atmospheric Chemistry and Physics, 14 (7), 3195-3210.

Pinel, J., Lovejoy, S., \& Schertzer, D. (2014). The horizontal space-time scaling and cascade structure of the atmosphere and satellite radiances. Atmospheric Research, 140, 95114.

Pope, S. B. (2001). Turbulent flows. IOP Publishing.

Ribal, A., \& Young, I. R. (2019). 33 years of globally calibrated wave height and wind speed data based on altimeter observations. Scientific Data, 6(1), 1-15.

Richardson, L. R. (1922). Weather prediction by numerical process. Cambridge University Press.

Schertzer, D., \& Lovejoy, S. (1985). Generalised scale invariance in turbulent phenomena. Physico Chemical Hydrodynamics, 6, 623-635.

Schertzer, D., \& Lovejoy, S. (1987). Physical modeling and analysis of rain and clouds by anisotropic scaling multiplicative processes. Journal of Geophysical Research: Atmospheres, 92(D8), 9693-9714.

Schlosser, E., Haumann, F. A., \& Raphael, M. N. (2018). Atmospheric influences on the anomalous 2016 Antarctic sea ice decay. The Cryosphere, 12(3), 1103-1119.

Schmitt, F. G. (2007). Direct test of a nonlinear constitutive equation for simple turbulent shear flows using DNS data. Communications in Nonlinear Science and Numerical Simulation, 12(7), 1251-1264.

Schmitt, F. G., \& Huang, Y. (2016). Stochastic analysis of scaling time series: from turbulence theory to applications. Cambridge University Press.

Spencer, M. W., Wu, C., \& Long, D. G. (1997). Tradeoffs in the design of a spaceborne scanning pencil beam scatterometer: Application to seawinds. IEEE Transactions on Geoscience and Remote Sensing, 35(1), 115-126.

Sreenivasan, K. R., \& Antonia, R. (1997). The phenomenology of small-scale turbulence. Annual Review of Fluid Mechanics, 29(1), 435-472.

Suquet, R. R., Hermozo, L., Tourain, C., Tison, C., Hauser, D., Schippers, P., ... Lachiver, J. M. (2019). CAL/VAL phase for the SWIM instrument onboard CFOSAT. In IGARSS 2019-2019 IEEE International Geoscience and Remote Sensing Symposium (pp. 8015-8018).

Tourain, C., Piras, F., Ollivier, A., Hauser, D., Poisson, J.-C., Boy, F., ... Tison, C. (2021). Benefits of the Adaptive algorithm for retracking altimeter nadir echoes: results from simulations and CFOSAT/SWIM observations. IEEE Transactions on Geoscience and Remote Sensing, 59, 1-14.

Tournadre, J. (1993). Time and space scales of significant wave heights. Journal of Geophysical Research: Oceans, 98(C3), 4727-4738.

Vallis, G. K. (2017). Atmospheric and oceanic fluid dynamics. Cambridge University Press.

Wikle, C. K., Milliff, R. F., \& Large, W. G. (1999). Surface wind variability on spatial scales from 1 to $1000 \mathrm{~km}$ observed during TOGA COARE. Journal of the Atmospheric Sciences, 56(13), 2222-2231.

Wyngaard, J. C. (2010). Turbulence in the atmosphere. Cambridge University Press.

$\mathrm{Xu}, \mathrm{Y} ., \mathrm{Fu}, \mathrm{L} .-\mathrm{L} .$, \& Tulloch, R. (2011). The global characteristics of the wavenumber spectrum of ocean surface wind. Journal of Physical Oceanography, 41(8), 1576-1582.

Xu, Y., Liu, J., Xie, L., Sun, C., Liu, J., Li, J., \& Xian, D. (2019). China-France Oceanography Satellite (CFOSAT) simultaneously observes the typhoon-induced wind and wave fields. Acta Oceanologica Sinica, 38(11), 158-161.

Zhang, K., Dong, X., Zhu, D., \& Yun, R. (2021). Estimation and correction of geolocation errors of the CFOSAT scatterometer using coastline backscatter coefficients. IEEE 
829

830

Journal of Selected Topics in Applied Earth Observations and Remote Sensing, 14, 53-61. 